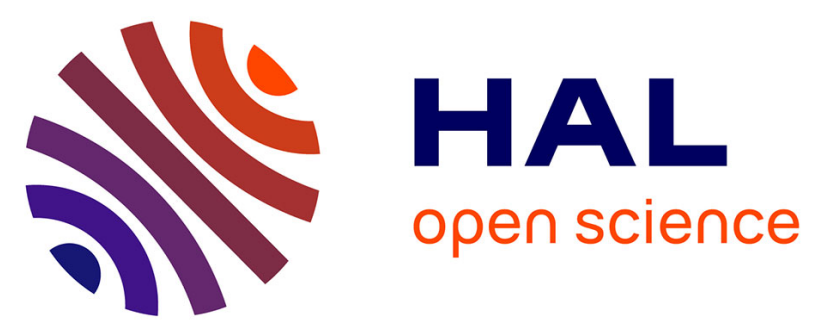

\title{
First Quantitative Assessment of the Adsorption of a Fluorocarbon Gas on Phospholipid Monolayers at the Air/Water Interface
}

\author{
Marie Pierre Krafft, Xianhe Liu, Claire Counil, Da Shi, Estefania E \\ Mendoza-Ortega, Andrea V Vela-Gonzalez, Armando Maestro, Richard A \\ Campbell, Marie Pierre Krafft
}

\section{To cite this version:}

Marie Pierre Krafft, Xianhe Liu, Claire Counil, Da Shi, Estefania E Mendoza-Ortega, et al.. First Quantitative Assessment of the Adsorption of a Fluorocarbon Gas on Phospholipid Monolayers at the Air/Water Interface. Journal of Colloid and Interface Science, 2021. hal-03175401

\author{
HAL Id: hal-03175401 \\ https://hal.science/hal-03175401
}

Submitted on 20 Mar 2021

HAL is a multi-disciplinary open access archive for the deposit and dissemination of scientific research documents, whether they are published or not. The documents may come from teaching and research institutions in France or abroad, or from public or private research centers.
L'archive ouverte pluridisciplinaire HAL, est destinée au dépôt et à la diffusion de documents scientifiques de niveau recherche, publiés ou non, émanant des établissements d'enseignement et de recherche français ou étrangers, des laboratoires publics ou privés. 
1 First Quantitative Assessment of the Adsorption of a Fluorocarbon Gas on

2 Phospholipid Monolayers at the Air/Water Interface

4 Xianhe Liu, ${ }^{\text {a }}$ Claire Counil, ${ }^{\text {a }}$ Da Shi, ${ }^{a}$ Estefania E. Mendoza-Ortega, ${ }^{a}$ Andrea V. Vela-Gonzalez, ${ }^{\text {a }}$

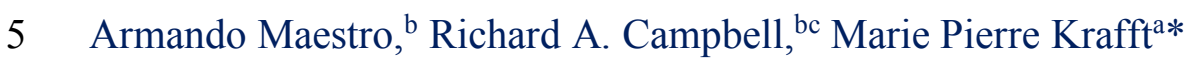

6

7 aUniversity of Strasbourg, Institut Charles Sadron (CNRS), 23 rue du Loess, 67034 Strasbourg Cedex, 8 France

9 bInstitut Laue-Langevin, 71 Avenue des Martyrs, CS20156, 38042 Grenoble Cedex 9, France

10 'Division of Pharmacy and Optometry, University of Manchester, Manchester M13 9PT, United 11 Kingdom

12

$13 *$ Corresponding author:

14 Dr. Marie Pierre Krafft

15 E-mail: $\underline{\text { krafft@,unistra.fr }}$

16 Tel: +33388414060

17

18

19 Keywords: DPPC; perfluorocarbon; neutron reflectometry; ellipsometry; mixed monolayers; isotopic 20 contrast 
1 ABSTRACT

2 Hypothesis: Fluorocarbon gases introduced above monolayers of phospholipids at the air/water

3 interface were recently found to promote the adsorption of diverse molecular compounds, with

4 potential application in drug-loaded microbubble design. Quantitative determination of the

5 fluorocarbon present in the monolayers is strongly needed for the development of such applications.

6 We hypothesized that neutron reflectometry (NR) and ellipsometry experiments would allow

7 quantification of the fluorocarbon trapped in the monolayers.

8 Experiments: We report the first quantitative determination of the extents of adsorption of

9 perfluorohexane ( $F$-hexane) on different phospholipid monolayers with respect to both their phase

10 and isotopic form. To this aim, we applied an approach based on co-modeling the data obtained from

$11 \quad$ NR and ellipsometry.

12 Findings: We found that $F$-hexane adsorbs strongly in monolayers of 13 dipalmitoylphosphatidylcholine (DPPC) when they are both in the liquid expanded (LE) and liquid 14 condensed (LC) phases, but to different extents according to the isotopic form of the phospholipid. 15 Kinetic resolution of the interfacial composition from data on both isotopic contrasts (assuming 16 chemical identicality) was therefore not possible using NR alone, so an alternative NR/ellipsometry 17 co-modeling treatment was applied to data from each isotopic contrast. $F$-hexane adsorbs more abundantly on monolayers of hydrogenous DPPC than chain-deuterated DPPC when they are in the LE phase, whilst the opposite was observed when they monolayers are in the LC phase. The extents of adsorption of $F$-hexane in monolayers of dimyristoylphosphatidylcholine (DMPC, LE phase) and

21 distearoylphosphatidylcholine (DSPC, LC phase) concurs with the strong dependence of those with 22 phospholipids of different isotopic contrasts according to the monolayer phase. This new methodology can lead to advances in the novel characterization of fluorocarbons interacting with phospholipid monolayers of relevance to applications such as in the shells of fluorocarbon-stabilized medically-oriented microbubbles. 


\section{1. INTRODUCTION}

2 Several products involving fluorocarbons (FCs), such as microbubbles and vaporizable 3 nanoemulsions, are under clinical evaluation for diverse applications in diagnostic and therapy, 4 including ultrasound imaging modes, non-invasive surgical tissue ablation, sonothrombolysis, 5 potentiation of $\mathrm{O}_{2}$-dependant cancer treatments such as radio-, chemo- and immunotherapy, blood-

6 brain barrier opening for treatment of central nervous system diseases, etc.[1-7] The FC-based colloidal systems most extensively investigated for medical uses comprise $F C$ nanoemulsions, nanoemulsions of volatile $F C$ that can be converted in microbubbles under various stimuli, and $F \mathrm{C}$ biophysical interactions, quantification of the composition of interfacial layers formed in the presence

11 of FCs, in particular the amount of fluorocarbon that becomes incorporated in the phospholipid 12 monolayer with respect to the phospholipid chain length and hence its phase, has not been achieved 13 to date. Fluorocarbon gases, when introduced in the gas phase above dipalmitoylphosphatidylcholine 14 (DPPC) monolayers, penetrate these monolayers and inhibit their liquid expanded (LE) to liquid condensed (LC) phase transition, that is, the formation of quasi-crystalline LC domains. [8, 9] The FC 16 gas can thus restore the re-spreading ability of DPPC molecules at the gas/water interface, enabling their use as active components of lung surfactant replacements.[8] Perfluorohexane (F-hexane) has also been shown to induce or accelerate the adsorption at the air/water interface of a range of molecular compounds, including lipids,[10] block co-polymers,[11] proteins,[12] cell biomarkers,[13] cerium oxide,[14] iron oxide nanoparticles,[15] and diamond [16] nanoparticles.

21 This attracting effect of $F$-hexane allowed the recruitment and immobilization of fluorinated cell 22 hypoxia biomarkers[13] and magnetic nanoparticles[15] in the phospholipid shell of microbubbles through fluorine-fluorine hydrophobic forces, without need for covalent binding, thus providing a

24 potential means for delivering the biomarker or nanoparticles through their ultrasound-mediated destruction. 
1 Monolayers of phospholipids, self-supported at the air/water interface, are effective models for

2 investigating the recognition, recruitment, and organization of biological and therapeutic water 3 soluble molecules at interfaces.[17-19] Planar air/water interfaces are also convenient platforms for 4 the application of various techniques, including spectroscopy,[20] rheology,[21] 5 electrochemistry[22] and scattering,[23] and interactions in phospholipid monolayers have been 6 investigated as a function of concentration,[24] $\mathrm{pH}[25]$ or temperature.[26] Specular neutron 7 reflectometry (NR) has long been recognized as an effective tool for investigating the structure and composition of mixed layers adsorbed at interfaces.[19, 27-29] This method typically requires corefined fitting of data acquired with different isotopic contrasts to a common structural model, an approach that usually requires deuterated compounds.

11 Recent advances in instrumentation have led to the provision of much higher neutron flux than was previously available,[30] in particular at low values of the momentum transfer normal to a reflecting air/water interface $\left(Q_{z}\right)$.[30] This new capability has opened up the quantitative real-time routine monitoring of adsorption kinetics and other dynamic processes in synthetic and biologicallyrelated systems.[31] A recently developed approach involving two parallel neutron reflectivity measurements only at low $Q_{\mathrm{z}}$ values allows one to solve the composition of a binary mixture much faster and more accurately than was formerly possible.[32, 33] An alternative approach to quantify the composition of a binary mixture, but much less exploited, consists of co-modeling data obtained using a single isotopic contrast of NR with data obtained by ellipsometry.[34] To the best of our knowledge, there has been no attempt yet to quantify the adsorption of fluorocarbons on phospholipid monolayers at the air/water interface. Indeed, even though NR is used routinely to investigate interactions of species in the bulk solution with phospholipid monolayers, $[35,36]$ and has been used to examine the effects on monolayers at the air/water interface from gas phase oxidants, $[37,38]$ there appears to have been no attempt yet to quantify the adsorption of molecules from the gas phase on monolayers at the air/water interface. 
1 Our goal in this work is to resolve quantitatively the interactions of fluorocarbon gases with planar model phospholipid membranes present in different phases at the air/water interface, which is an essential step to understanding and developing the potential of FCs as reinforcers of microbubble shells for diagnostics and therapy or as components of lung surfactant substitutes. As the low- $Q_{z}$ method of NR to quantify the interfacial composition of binary mixtures at the air/water interface requires parallel measurements involving components in their native and deuterated forms, and as $F$ hexane has no hydrogen protons, we have performed NR and ellipsometry measurements using hydrogenous DPPC (h-DPPC) and its isotopic analogue bearing $\mathrm{d}_{62}$-perdeuterated acyl chains (dDPPC), both in the LE and LC phases. NR and ellipsometry data were combined in order to determine the surface excesses of DPPC and $F$-hexane. This study is the first quantitative investigation of the adsorption and incorporation of a fluorocarbon in phospholipid monolayers and also, to our knowledge, the first quantitative assessment of a multi-component system involving adsorption of a component originating from the gas phase.

The manuscript is organized as follows. The principles of NR and ellipsometry are briefly presented in Section II. In Section III, results are presented and discussed: analysis of ellipsometry data recorded in different isotopic contrasts of the same systems reveals that $F$-hexane interacts differently with h- and d-DPPC (III.1); quantitative analysis of NR data of $F$-hexane with h- and dDPPC monolayers using a standard approach confirms that the assumption of equivalent physical interactions of $F$-hexane with the DPPC isotopic forms is unjustified for this system (III.2); comodeling NR and ellipsometry data enables quantification of the interfacial composition in both $F$ hexane/h-DPPC and F-hexane/d-DPPC mixed monolayers (III.3); in Section IV, adjustment coefficients $R$ that were determined for DPPC monolayers in various phases are applied to monolayers of two other phospholipids, dimyristoylphosphatidylcholine (DMPC), which is in the LE phase, and distearoylphosphatidylcholine (DSPC), which is in the LC phase. The results extend our quantification method to different lipid systems while further validating our approach that combines NR and ellipsometry. 
1 II. MATERIALS AND METHODS

\section{Materials}

1,2-Dimyristoylphosphatidylcholine (h-DMPC), 1,2-dipalmitoylphosphatidylcholine (h-DPPC), 1,2-distearoylphosphatidylcholine (h-DSPC), all $>99 \%$, and their chain-deuterated analogues $\left(\mathrm{d}_{54-}\right.$ DMPC; $\mathrm{d}_{62}$-DPPC; and $\mathrm{d}_{70}$-DSPC, all >99\%) were purchased from Avanti Polar Lipids (Alabaster, AL, USA) and used without further purification. Perfluorohexane came from Fluorochem ( $>98 \%)$. A Hepes (N-2-(hydroxyethyl)piperazine-N'-(2-ethanesulfonic acid), powder, 99.5\%, Corning, NY) buffer $(20 \mathrm{mM})$ in $150 \mathrm{mM} \mathrm{NaCl}$ was prepared and adjusted to $\mathrm{pH} 7.4$ using $0.1 \mathrm{~N} \mathrm{NaOH}$. Chloroform (99.4\%) was purchased from VWR (Avantor, Fontenay-sous-Bois). Ultrapure water was obtained from a Milli-Q (Millipore Corp.) system (surface tension: $72.1 \mathrm{mN} \mathrm{m}^{-1}$ at $20^{\circ} \mathrm{C}$, resistivity: $18.2 \mathrm{M} \Omega$ $\mathrm{cm}$ ). For the NR experiments, the Hepes buffer was prepared in air contrast matched water (ACMW, containing $8.1 \%$ by volume of $\mathrm{D}_{2} \mathrm{O}$ (Sigma Aldrich, $>99.9 \%$ D) in $\mathrm{H}_{2} \mathrm{O}$ ).

\section{Ellipsometry}

Ellipsometry is a fast and precise polarized optical reflectometry technique for the characterization of thin films (the film thickness should be smaller than the laser wavelength in the Drude approximation) and the kinetics of adsorption processes. $[39,40]$ Its footprint is $<1 \mathrm{~mm}^{2}$, as compared to several $\mathrm{cm}^{2}$ for NR. Although ellipsometry does not allow direct determination of the surface excesses of multiple components at the interface, it has been used along with NR to perform such quantitative analysis.[34] Ellipsometry measures the change of polarization that light undergoes when it is reflected at an interface. The polarization change is quantified by an amplitude ratio $\Psi$ and a phase shift $\Delta$ that are related to the reflection coefficients (parallel $r_{\mathrm{p}}$ and perpendicular $r_{\mathrm{s}}$ to the plane of incidence) by the relation: $r_{\mathrm{p}} / r_{\mathrm{s}}=\tan \Psi \mathrm{e}^{\mathrm{i} \Delta}$. As $\Psi$ is relatively insensitive to thin layers present at the air/water interface, the quantity measured in the present work is the change in phase shift at the interface $\Delta=\Delta_{\text {meas }}-\Delta_{0}$, where $\Delta_{\text {meas }}$ is the value measured after deposition of the phospholipid in the presence, or absence, of the fluorocarbon, and $\Delta_{0}$ the value for the bare air/water 
1 interface. This subtraction process approximately eliminates the effects of capillary wave roughness,[41] neglecting the relatively minor influence of the surface tension change from sample to sample. The surface excess $\Gamma$ can be considered proportional to $\Delta$ for layers at the air/water interface in the thin film limit, provided that the thickness increases with $\Gamma$ with a uniform density 5 (i.e. oil-like behavior).[42] For hydrocarbon surfactants, the relationship between $\Gamma$ and $\Delta$ is approximatively linear.[34] In some cases, however, the linearity is no longer valid, which include anisotropy [43, 44], layers at the air/water interface in the thin film limit where with increasing surface excess the density increases at uniform thickness,[40] and films thicker than $\sim 10 \mathrm{~nm}$.[39] In the present work, we assume linearity of the $\Gamma(\Delta)$ relationship for fluorocarbon/phospholipid systems in a given phase, as the condensation of the fluorocarbon on a monolayer is expected to result in an increase in thickness rather than density, and we follow the approximation that $\Delta$ is an additive function of the amounts of adsorbed compounds.[34] Thus, the phase shift $\Delta_{x}$ of a single component variation. area $\sim 30 \mathrm{~cm}^{2}$ ) enclosed in a chamber designed at the ILL.[46] The concentrations of the DPPC solutions were the same as those used in the NR measurements, and the dispensed volumes were 
1 as in NR. The experiments were repeated at least three times. The experimental errors on $\Delta$

2 measurements were $\pm 2 \%$ for phospholipids exposed to air or $F$-hexane; and $\pm 3 \%$ for experiments

3 involving $F$-hexane alone.

\section{$43 . \quad$ Neutron Reflectometry}

a. Principles of low- $Q_{\mathrm{z}}$ compositional analysis

NR is a powerful tool for the investigation of thin films[47], including phospholipid monolayers, due to the possibility of exploiting isotopic contrast variation.[28, 48-52] The use of ACMW, which has the same neutron scattering length density as air, enables the specular reflectivity signal to arise only from any layer at the interface. The neutron reflectivity profiles show the intensity ratio of neutrons in the specular reflection from the sample to those in the incident beam with respect to the momentum transfer $Q_{z}$, as defined by $Q_{z}=(4 \pi \sin \theta) / \lambda$. NR profiles can be modeled to obtain information about the structure and composition of an interfacial layer from the fitted thickness $d$ and scattering length density $\rho$. However, in our case, the NR data were deliberately acquired only on a restricted $Q_{z}$ range $\left(0.01-0.03 \AA^{-1}\right)$ and the surface excess of each component at the interface was determined using the low- $Q_{\mathrm{z}}$ compositional analysis method.

In a single component monolayer at the air/water interface, the surface excess is given by $\Gamma_{x}=$ $\frac{\rho d}{b_{x} \mathrm{~N}_{\mathrm{A}}}$, where $b_{x}$ is the scattering length of component $x$ and $\mathrm{N}_{\mathrm{A}}$ is Avogadro's constant, $\rho$ is the scattering length density of the component, $d$ is the thickness of the layer. The so-called "scattering excess" of the layer at low $Q_{z}$, which is expressed in terms of the amount of interfacial component with respect to its scattering contrast, is thus described as $(\rho d)=\mathrm{N}_{\mathrm{A}} \Gamma_{\mathrm{x}} b_{\mathrm{x}}$. The scattering excess of

21 a mixed layer at low $Q_{\mathrm{z}}$, expressed in terms of the amount of each interfacial component with respect to its scattering contrast, may be described as $(\rho d)=\mathrm{N}_{\mathrm{A}} \sum \Gamma_{\mathrm{x}} b_{\mathrm{x}} \cdot$ [28] This approach is based on the approximation that the scattering contributions of each component is additive as well as the assumption that the interfacial material can be modeled as a single uniform layer, i.e., low- $Q_{z}$ nature 
1 normal to the interface. This approximation and assumption are scrutinized below. The reason that this approach was used is that the traditional, structural analysis of NR data over the whole $Q_{\mathrm{z}}$-range remains too slow currently to resolve the interaction kinetics of interest.

b. Experimental Set-Up

NR measurements were performed using FIGARO (Fluid Interface Grazing Angle ReflectOmeter) reflectometer optimized for horizontal surfaces at the Institut Laue-Langevin (ILL, Grenoble, France).[30] A beam of neutrons with $\lambda=2-16 \AA$ impinged upon the samples at $\theta=0.62^{\circ}$, although a restricted range of $\lambda=4.5-12 \AA$ was used in the data reduction process to generate reflectivity profiles with a $Q_{\mathrm{z}}$ range of $0.01-0.03 \AA^{-1}$ at a resolution of $8 \%$. The acquisition time for each sample was $5 \mathrm{~min}$. The data analysis was performed using an arbitrary scattering length density $11 \rho$ of $5 \times 10^{-6} \AA^{-2}$ with the Motofit program in the Igor software, which allows to obtain the thickness value $d$.[53] An investigation showed that fitting with refined $\rho$ values instead of the arbitrary value led to small difference $(<3 \%)$ in the calculation of the surface excesses of DPPC and $F$-hexane. (see SI "Fitting parameter in low- $Q_{z}$ analysis", Table S1). It was also verified that our treatment of the data was valid for layer thicknesses lower than $60 \AA$ (see SI, Fig. S1, Table S3, Scheme S3 and Table S8). The layer roughness was set to $3 \AA$. Six adsorption troughs with sealed lids constructed at the solutions $(18 \mathrm{~mL})$ were deposited in each trough prior to the deposition of a $1-\mathrm{mM}$ solution of the phospholipid (DPPC, DMPC or DSPC) in $\mathrm{CHCl}_{3}$. The gas phase above the monolayers consisted either of air, or of air saturated with $F$-hexane. In the latter case, an open flat aluminum dish $(5.5 \times$ $8.8 \times 0.5 \mathrm{~cm}^{3}$ ) was filled with $20 \mathrm{~mL}$ of liquid $F$-hexane and introduced next to each trough. The measurements were all conducted at $21 \pm 1{ }^{\circ} \mathrm{C}$. The saturated vapor pressure and concentration of $F$ hexane at $25^{\circ} \mathrm{C}$ are $2.9 \times 10^{4} \mathrm{~Pa}\left(2.3 \times 10^{4} \mathrm{~Pa}\right.$ at $\left.21^{\circ} \mathrm{C}\right)$ and $11.66 \mathrm{~mol} \mathrm{~m}^{-3}$, respectively; its water solubility is estimated at $2.7 \times 10^{-4} \mathrm{~mol} \mathrm{~m}^{-3}$ at $25^{\circ} \mathrm{C}$. [54-56] The saturation of the atmosphere in the trough was confirmed for each experiment by the presence of some liquid $F$-hexane still left in the dish when the lids were removed. 
1 Because the surface pressure $\pi$ could not be measured in the adsorption troughs during NR measurements, we determined in advance of the neutron experiment the variation of $\pi$ as a function of the DPPC spread volume, under air or $F$-hexane exposure in a trough similar to those used on FIGARO and at the same temperature $\left(21 \pm 1^{\circ} \mathrm{C}, \mathrm{SI}\right.$, Fig. S2a). This procedure allowed reconstruction of the $\pi / A$ isotherms for DPPC monolayers investigated during the NR experiments (SI, Fig. S2b). Various volumes of DPPC solution were deposited in order to provide monolayers in the LE phase ( 8 and $10 \mu \mathrm{L})$, in the LE-LC coexistence phase (12 and $14 \mu \mathrm{L})$ and in the LC phase $(16 \mu \mathrm{L})(\mathrm{SI}$, Table S4). It was verified that h- and d-DPPC monolayers were in comparable physical state under air and under $F$-hexane exposure for the same deposited volume, hence same molecular area (SI, Fig. S3a). Appropriate spread volumes of DMPC solutions in $\mathrm{CHCl}_{3}(15 \mu \mathrm{L})$ or DSPC $(26 \mu \mathrm{L})$ provided monolayers at $35 \mathrm{mN} \mathrm{m}^{-1}$, which corresponds to the LE phase for DMPC and to the LC phase for DSPC (SI, Fig. S3b,c). The lids of the troughs were closed and the NR measurements were conducted for $6 \mathrm{~h}$. For the experiments in which $F$-hexane was present, the lids were opened after the $6 \mathrm{~h}$-period of monitoring in order to vent $F$-hexane out of the system. The measurements were then resumed for another $2 \mathrm{~h}$ period. Neutron reflectivity profiles of the adsorption of $F$-hexane at the surface of the Hepes buffer were also measured in the absence of phospholipid. The experiments were repeated at least three times. Experimental errors on the surface excesses of DPPC $\left(\Gamma_{\text {DPPC }}\right)$ are estimated $\leq 7 \%$ $\left( \pm 0.1 \mu \mathrm{mol} \mathrm{m} \mathrm{m}^{-2}\right.$ for LE phase and $\pm 0.2 \mu \mathrm{mol} \mathrm{m} \mathrm{m}^{-2}$ for LC phase), and $\sim 6 \%\left( \pm 1.0 \mu \mathrm{mol} \mathrm{m} \mathrm{m}^{-2}\right.$ ) for $F$ hexane $\left(\Gamma_{F-\text { hex }}\right)$.

\section{RESULTS AND DISCUSSION}

\section{Ellipsometry Reveals a Difference in $F$-hexane Interactions with h-DPPC and d-DPPC}

Ellipsometry was initially used in order to examine two isotopic forms of DPPC monolayer both in air and when exposed to $F$-hexane saturated air. The variation of the phase shift $\Delta_{\text {meas }}$ was monitored over time for monolayers of h- and d-DPPC in the LE and LC phases and under air or $F$ hexane-saturated air (Fig. 1ab). The adsorption of $F$-hexane on the surface of the aqueous phase (i.e. 
1 in the absence of phospholipid) was also monitored (Fig. 1c). In air (Fig. 1a), we observe that 1) $\Delta_{\text {meas }}$

2 is higher for DPPC monolayers in the LC than in the LE phase, which is expected because the surface

3 excess $\Gamma$ is higher (spread values correspond to $\Gamma=2.8$ vs. $1.7 \mu \mathrm{mol} \mathrm{m}^{-2}$ for the LC and LE phases,

4 respectively); 2) $\Delta_{\text {meas }}$ does not exhibit a rising or falling trend over time; and 3) $\Delta_{\text {meas }}$ depends only

5 slightly on the DPPC isotopic form $\left(2.26 \pm 0.05^{\circ}\right.$ and $2.19 \pm 0.05^{\circ}$ for h- and d-DPPC in the LE phase;

6 and $3.03 \pm 0.05^{\circ}$ and $2.82 \pm 0.05^{\circ}$ in the LC phase). By contrast, when the DPPC monolayers are

7 exposed to $F$-hexane (Fig. 1b), two significant differences are observed. First, $\Delta_{\text {meas }}$ is no longer

8 constant over time as its adsorption is progressive. This can result from the fact that the adsorption of

$9 \quad F$-hexane decreases the value of $\Delta$ because $F$-hexane has a smaller refractive index than water $(1.251$

10 [57] vs. 1.333; Fig. 1c). Second, $\Delta_{\text {meas }}$ is no longer the same for the two isotopic forms. In both LE

11 and LC states, $\Delta_{\text {meas }}$ is lower for d-DPPC than for h-DPPC throughout the adsorption time period

12 (Fig. 1b). In the LE phase, the $\Delta_{\text {meas }}$ minimum values were $1.32^{\circ}$ and $1.87^{\circ}$ for d-DPPC and h-DPPC,

13 respectively (i.e. a difference of $0.55^{\circ}$ ), indicating higher adsorption of $F$-hexane on d-DPPC

14 monolayer. In the LC phase, the difference was lower $\left(0.27^{\circ}\right.$; with $\Delta$ minimum values of $2.29^{\circ}$ and

$152.02^{\circ}$ for d-DPPC and h-DPPC, respectively).

16

17

18

19

20

21
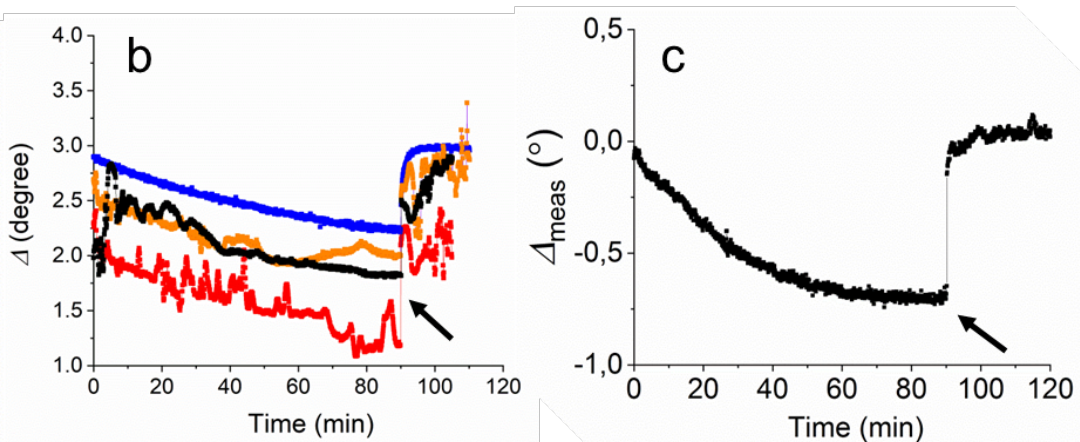

Figure 1. Variation of the phase shift $\Delta_{\text {meas }}$ over time, as measured by ellipsometry for DPPC monolayers in the LE phase (black: h-DPPC; red: d-DPPC) and LC phase (blue: h-DPPC; orange: dDPPC) under a) air and b) $F$-hexane exposure. c) Variation of $\Delta_{\text {meas }}$ over time for $F$-hexane adsorption on the aqueous phase in the absence of phospholipid. The arrows indicate the time point at which $F$ hexane was vented-off. The experimental errors were $\pm 2 \%$ for DPPC monolayers exposed to air or to $F$-hexane; and $\pm 3 \%$ for $F$-hexane adsorbing at the surface of the aqueous phase. 
1 It is known that the difference between the refractive indices of $\mathrm{h}$ - and d-hydrocarbons decreases

2 when their carbon atom number increases, [58] thus resulting in a strong decrease of the proportional

3 change in $\Delta n=n-n_{\text {water }}$ ( $n$ and $n_{\text {water }}$ being the refractive indices of a given hydrocarbon and water,

4 respectively) for h-and d-hydrocarbons. It is likely that for long hydrocarbon chains such as those of

5 DPPC, this proportional change becomes negligible. As a consequence, any difference in the

6 ellipsometry data can be attributed to different extents of interaction of $F$-hexane to h- and d-DPPC.

7 These results show that isotopic effects are observed for DPPC monolayers in LE and LC states, with

$8 \quad F$-hexane interacting more strongly with d-DPPC than with h-DPPC. It is noted that when $F$-hexane

9 is vented-off from the adsorption trough, $\Delta_{\text {meas }}$ returns back to its initial value, that is, to that measured in the absence of the fluorocarbon, which demonstrates that the DPPC/F-hexane interactions are

11 reversible. Quantification of these effects was needed, as described in Section 3.

\section{Quantitative Analysis by Neutron Reflectometry Confirms the Difference in $\boldsymbol{F}$-hexane}

\section{Interactions with h-DPPC and d-DPPC}

To confirm and explore the implication of the isotope effects suggested by the ellipsometry data presented above, neutron reflectivity profiles of h-DPPC and d-DPPC monolayers were measured in air and under $F$-hexane exposure in the low- $Q_{z}$ range $\left(0.01-0.03 \AA^{-1}\right)$ (Fig. 2).
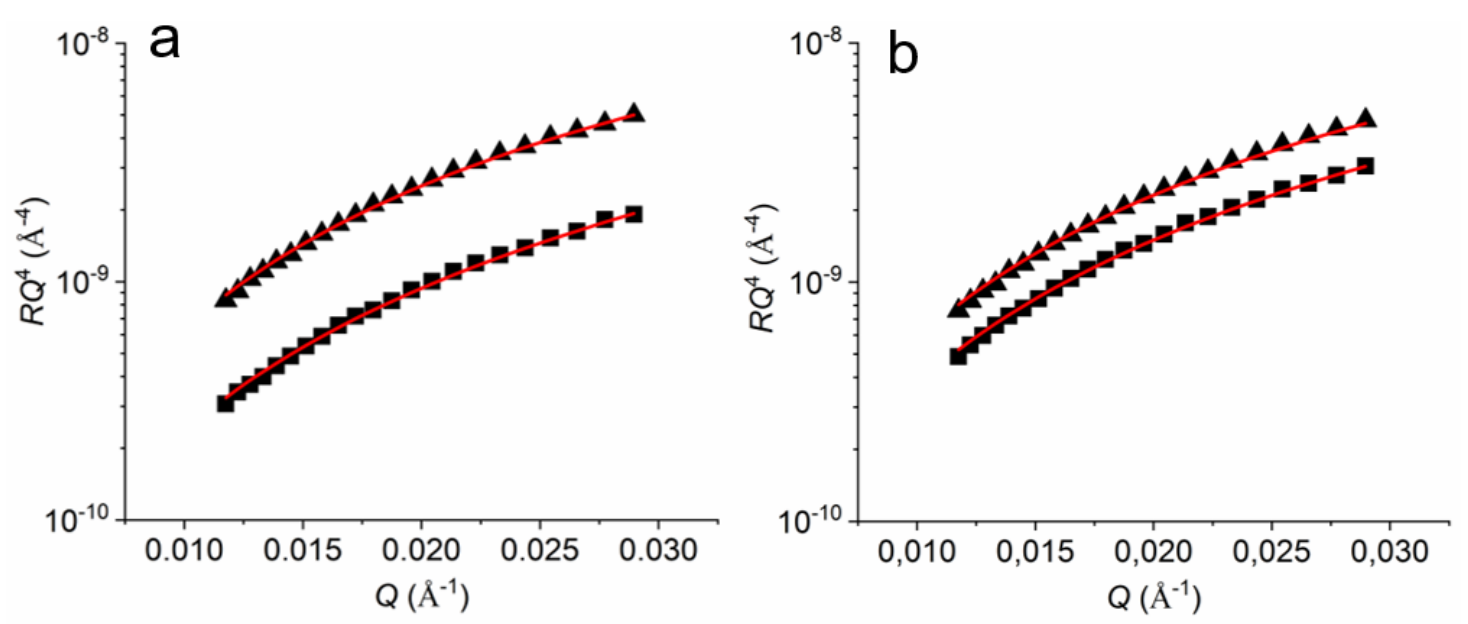

Figure 2. Measured (h-DPPC: squares; d-DPPC: triangles) and fitted (red line) variation of $R Q^{4}$ as a function of $Q_{z}$ of DPPC monolayers exposed to $F$-hexane a) in the LE phase and $\mathrm{b}$ ) in the LC phase at the adsorption equilibrium (at $\sim 4 \mathrm{~h}$ ). 
1 First, for reference, the data were modeled using a standard treatment, that is, assuming that $F$ -

2 hexane interacts to the same extent with h- and d-DPPC monolayers, and that the resulting layers are

3 homogeneous. The surface excesses of h-DPPC $\left(\Gamma_{\mathrm{h}-\mathrm{DPPC}}\right)$ and d-DPPC $\left(\Gamma_{\mathrm{d}-\mathrm{DPPC}}\right)$ and the surface

4 excesses of $F$-hexane when interacting with h-DPPC and d-DPPC, $\left(\Gamma_{F \text {-hex } / \mathrm{h} \text {-DPPC }}\right.$ and $\left.\Gamma_{F \text {-hex } / \mathrm{d} \text {-DPPC }}\right)$

5 are given in the following equations:

$(\rho d)_{\mathrm{h}-\mathrm{DPPC}}^{F-\mathrm{hex}}=N_{\mathrm{A}}\left(\Gamma_{\mathrm{h}-\mathrm{DPPC}} \times b_{\mathrm{h}-\mathrm{DPPC}}+\Gamma_{F \text {-hex } / \mathrm{h} \text {-DPPC }} \times b_{F \text {-hex }}\right) \quad$ Eq. 1

$(\rho d)_{\mathrm{d}-\mathrm{DPPC}}^{F-\text { hex }}=N_{\mathrm{A}}\left(\Gamma_{\mathrm{d} \text {-DPPC }} \times b_{\mathrm{d} \text {-DPPC }}+\Gamma_{F-\text { hex } / \mathrm{d}-\mathrm{DPPC}} \times b_{F-\text { hex }}\right)$

Eq. 2

8 where $(\rho d)_{\mathrm{h}-\mathrm{DPPC}}^{F-\text { hex. }}$ and $(\rho d)_{\mathrm{d}-\mathrm{DPPC}}^{F-\text { hex. }}$ are the scattering excesses of the h- and d-DPPC monolayers exposed to $F$-hexane; $b_{\mathrm{h} \text {-DPPC }}, b_{\mathrm{d} \text {-DPPC }}$ and $b_{F \text {-hex }}$ are the scattering lengths of h-DPPC (27.55 fm), d-DPPC (673.03 fm) and $F$-hexane $(117.772 \mathrm{fm})$, respectively. Under air, $\Gamma_{\mathrm{DPPC}}$ is calculated with 11 equations 1 and 2 with $\Gamma_{F-\text { hex } / \mathrm{h}-\mathrm{DPPC}}=\Gamma_{F \text {-hex } / \mathrm{d} \text {-DPPC }}=0$. As $\Gamma_{\mathrm{h}-\mathrm{DPPC}}$ and $\Gamma_{\mathrm{d} \text {-DPPC }}$ are assumed to be equal (same quantities of h-DPPC and d-DPPC were deposited; noted $\Gamma_{\mathrm{DPPC}}$ below) and as $\Gamma_{F \text {-hex/h-DPPC }}$ and $\Gamma_{F \text {-hex/d-DPPC }}$ are also equal (our hypothesis), Eqs. $1 \& 2$ can be resolved to calculate $14 \Gamma_{\mathrm{DPPC}}$ and $\Gamma_{\mathrm{F}-\mathrm{hex}}$.

15 It is observed that for monolayers in the LE phase under $F$-hexane exposure (Fig. 3a), based on 16 the above assumptions, $\Gamma_{\mathrm{DPPC}}$ would be higher than the value measured under air $(2.1 \mathrm{vs} .1 .7 \mu \mathrm{mol}$ $17 \mathrm{~m}^{-2}, \pm 0.2 \mu \mathrm{mol} \mathrm{m} \mathrm{m}^{-2}$ ), and also higher than the value that corresponds to the amount of phospholipid 18 deposited $\left(1.7 \mu \mathrm{mol} \mathrm{m} \mathrm{m}^{-2}\right)$, which is physically unrealistic. In the LC phase (Fig. 3b), $\Gamma_{\mathrm{DPPC}}$ was found 19 to be lower than the value under air and the deposited value, a result that could be explained by a desorption of DPPC by $F$-hexane, which would be caused either by 3D collapse or solubilization of 21 phospholipid molecules in the sub-phase in the form of vesicles. The latter hypothesis is unlikely as 3D collapse of bilayers on top of a DPPC monolayer has never been observed in previous investigations by fluorescence microscopy or SAXS. $[59,60]$ On the contrary, all these previous investigations demonstrated that the $F C$ gas causes a fluidization of the DPPC monolayer, even at high surface pressure, and dissolution of DPPC liquid condensed phase domains.[59, 60] 

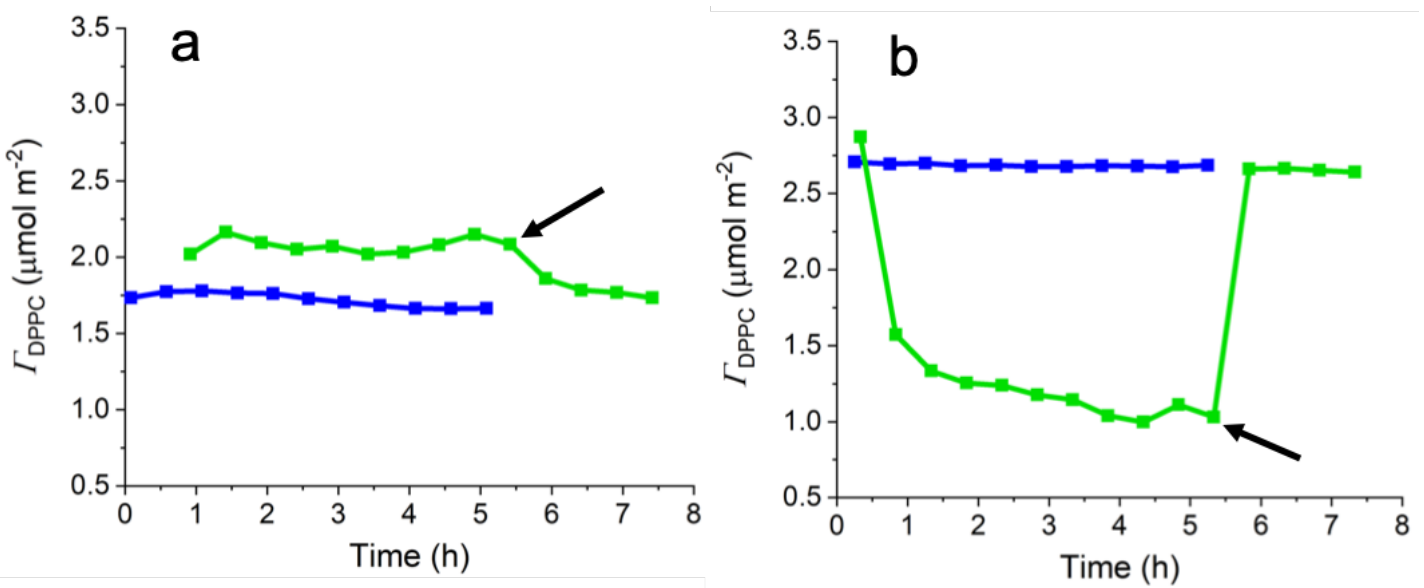

Figure 3. Variation of DPPC surface excess, $\Gamma_{\mathrm{DPPC}}$, over time for DPPC monolayers in a) the LE phase and $b$ ) the LC phase, under air (blue) and under $F$-hexane exposure (green). $\Gamma_{\mathrm{DPPC}}$ is calculated with the assumption that the interactions between $F$-hexane and DPPC isotopic forms are identical. The arrows indicate when $F$-hexane has been vented off from the system. Errors on $\Gamma_{\mathrm{DPPC}}$ were $\pm 0.2 \mu \mathrm{mol} \mathrm{m}^{-2}$.

Whilst it would be intuitive on the basis of the ellipsometry results above to attribute these physically unrealistic results to limitations of the analysis method applied in that $F$-hexane is interacting with h-DPPC and d-DPPC to different extents, we went on to scrutinize whether our assumption of modeling the low- $Q_{\mathrm{z}}$ data as a single, uniform layer could instead explain, or at least contribute, to the unphysical nature of the results above. The possible effects of non-uniformity in the direction normal to the interface (i.e. different locations of $F$-hexane either in a layer above or mixed in with the lipid chains, themselves in a layer above solvated headgroups), or non-uniformity in the direction lateral to the interface (i.e. the possibility of domains of $F$-hexane sitting in between regions of lipid monolayer on a length scale above the coherence length of the neutrons) were examined. These tests scrutinized the insensitivity to the interfacial structure of the low- $Q_{z}$ analysis method and indeed went beyond lengths taken to validate the approach in any such previous study. The tests were performed by simulating neutron reflectivity data of the mixed $F$-hexane/lipid systems in different isotopic contrasts using a structural model and parameters that had been validated using data recorded in 4 isotopic contrasts over the fully accessible $Q_{z}$-range,[33] before applying the low- $Q_{z}$ analysis method to the same restricted $Q_{\mathrm{z}}$-range using the same approach as described herein. More 
1 information can be found in the Supporting Information (SI p. S5 "Simulation of the Effect of Vertical

2 Separation" and p. S9 "Simulation of the Effect of Lateral Separation").

3 The results of the simulations revealed that a vertical separation of the scattering length density

4 profile resulting from stratification of the $F$-hexane (of different locations), lipid chains and solvated

5 lipid headgroups normal to the interface results in a maximum uncertainty in the resulting interfacial

6 composition of $3 \%$ for DPPC and $6 \%$ for $F$-hexane, and that use of a heterogeneous model to mimic

7 lateral separation made the unphysical nature of the resulting DPPC surface excess even more pronounced. These simulations also serve to validate the use of the low- $Q_{z}$ analysis method with a single, uniform layer model in our case, as there is no indication that a laterally-heterogeneous model is more appropriate, uncertainties introduced from the additivity approximation about the scattering

11 from different components are minimal, and fits to the experimental data were significantly poorer 12 when the lateral domain thickness of $F$-hexane was consistent with full lateral separation of the interfacial components.

14 We thus conclude that the physically unrealistic data above are not the result of vertical or lateral heterogeneity that would invalidate use of a single uniform layer in the low- $Q_{z}$ data analysis, but instead that $F$-hexane indeed interacts to different extents with the two isotopic forms of DPPC, as indicated by ellipsometry in Section 1. It is interesting to note that phospholipid monolayers do not generally exhibit strong isotopic effects, as lipophilic attraction between hydrocarbon chains is the main driving force of monolayer dynamics.[61] However, the interaction energies associated with $\mathrm{H}$ bonds and D-bonds are different, which has pronounced effect on the phase transitions in 21 phospholipid mono- and bilayers, $[48,62-65]$ and may help to explain the present results.

\section{Co-modeling Ellipsometry and NR Data}

In Section 2, we have observed that, owing to the difference in interactions of $F$-hexane with the two isotopic forms of DPPC, the low- $Q_{z}$ analysis method of NR alone - i.e. solving the surface excess of two components by making measurements in two different isotopic contrasts using Eqs 1 and 2 is not sufficient to resolve the interfacial composition of the system because the assumption that the 
1 interfacial composition is the same in the two measurements is not valid. An alternative approach to

2 resolve quantitatively the composition of a binary mixture is to co-model data from one NR

3 measurement in a single isotopic contrast and one ellipsometry measurement, as described by Bain

4 et al.[34]

5 We have applied this approach for NR and ellipsometry data recorded for h- and d-DPPC under

6 air and $F$-hexane-saturated air in order to treat the systems of different isotopic contrasts individually.

7 The phase shifts of $\mathrm{h}$ - and d-DPPC monolayers under $F$-hexane exposure, as measured by $8 \quad$ ellipsometry, are given by Eq. $3 \& 4$ :

9

10

11

12

13

14

15

16

17
$\Delta_{h-D P P C}^{F-h e x}=k_{\mathrm{h}-\mathrm{DPPC}} \times \Gamma_{\mathrm{h}-\mathrm{DPPC}}+k_{F-\mathrm{hex}} \times \Gamma_{F-\mathrm{hex} / \mathrm{h}-\mathrm{DPPC}}$

Eq. 3

$\Delta_{d-D P P C}^{F-h e x}=k_{\mathrm{d}-\mathrm{DPPC}} \times \Gamma_{\mathrm{d}-\mathrm{DPPC}}+k_{F-\mathrm{hex}} \times \Gamma_{F-\mathrm{hex} / \mathrm{d}-\mathrm{DPPC}}$

Eq. 4

where $\Delta_{h-D P P C}^{F-h e x}$ and $\Delta_{d-D P P C}^{F-h e x}$ are the phase shifts of h- and d-DPPC monolayers under $F$-hexane as measured by ellipsometry (average of values over $1 \mathrm{~h}$ taken after equilibrium reached after $1.5 \mathrm{~h}$ ). $k_{\mathrm{h}-\mathrm{DPPC}}, k_{\mathrm{d}-\mathrm{DPPC}}$ and $k_{F-\mathrm{hex}}$ are the slopes of the linear variations of $\Delta$ as a function of $\Gamma$, were obtained in the single-components systems using the equation $k_{x}=\Delta_{x} / \Gamma_{\mathrm{x}}$, where $\Delta_{x}$ was measured by ellipsometry and $\Gamma_{\mathrm{x}}$ by NR.

Results, collected in Table 1, show that the $k$ values are influenced mainly by the phase of the monolayer, LE versus LC, as reported earlier and ascribed to monolayer optical anisotropy.[43, 44, 66] On the other hand, the values are not much influenced by the isotopic form.

Table 1. Phase shift $\Delta$ (from ellipsometry) and surface excess $\Gamma$ (from NR) of hydrogenous and deuterated DPPC monolayers under air, and of an adsorbed layer of $F$-hexane in the absence of phospholipids; $k$ values are the slope of the linear variation of $\Delta$ as a function of $\Gamma$.

\begin{tabular}{l|ccc} 
& $\begin{array}{c}\text { Phase shift } \\
\Delta\left({ }^{\circ}\right)\end{array}$ & $\begin{array}{c}\text { Surface excess } \\
\Gamma\left(\mu \mathrm{mol} \mathrm{m}^{-2}\right)\end{array}$ & $k$ \\
\hline h-DPPC (LE) & $2.26 \pm 0.05$ & $1.6 \pm 0.1$ & 1.4 \\
d-DPPC (LE) & $2.19 \pm 0.05$ & & 1.4 \\
\hline h-DPPC (LC) & $3.03 \pm 0.05$ & $2.8 \pm 0.2$ & 1.1 \\
d-DPPC (LC) & $2.82 \pm 0.05$ & & 1.0 \\
\hline F-hexane & $-0.7 \pm 0.02$ & $19.0 \pm 1.0$ & -0.04
\end{tabular}


1 These $k$ values were used for the calculation of surface excesses in DPPC/F-hexane monolayers.

2 Co-modeling NR and ellipsometry data for $F$-hexane adsorption on h- and d-DPPC monolayers in

3 LE and LC phases, led to the values of $\Gamma_{\mathrm{h} \text {-DPPC }}, \Gamma_{\mathrm{d}-\mathrm{DPPC}}, \Gamma_{F-\mathrm{hex} / \mathrm{h}-\mathrm{DPPC}}$, and $\Gamma_{F \text {-hex/d-DPPC }}$ (using Eqs.

$41 \& 3$ for h- DPPC and Eqs. $2 \& 4$ for d-DPPC). It is seen in Figure 4a that, for each physical state,

$5 \quad \Gamma_{\mathrm{h}-\mathrm{DPPC}}$ and $\Gamma_{\mathrm{d} \text {-DPPC }}$ are approximately identical (difference within the experimental error), and constant over time, whether the DPPC monolayers are exposed to air or to $F$-hexane. This shows that the co-modeling method, independent of the isotopic effect, permits accurate calculation of DPPC surface excesses. The surface excesses correspond well to the quantities deposited, which endorses the fact that the extent of the interactions of $F$-hexane depends on the isotopic form. It is also observed that $F$-hexane always adsorbs strongly on DPPC monolayers. The surface excess of the fluorocarbon

11 is higher on d-DPPC than on h-DPPC monolayers in the LE phase (20.0 vs. $16.8 \mu \mathrm{mol} \mathrm{m}^{-2}$, 12 respectively; $\pm 1.0 \mu \mathrm{mol} \mathrm{m} \mathrm{m}^{-2}$, while it is lower in the LC phase (13.5 vs. $20.8 \mu \mathrm{mol} \mathrm{m}{ }^{-2}$, respectively, $13 \pm 1.0 \mu \mathrm{mol} \mathrm{m} \mathrm{m}^{-2}$ ) (Fig. 4b). This confirms that the interactions of the fluorocarbon are different with 14 the hydrogenous or deuterated phospholipid, and suggests that the extents of interaction are also 15 influenced by the phase of the DPPC monolayers. The adjustment coefficient $\Gamma_{F-\text { hex } / \mathrm{h} \text {-DPPC }} /$ $\Gamma_{F \text {-hex } / \text { d-DPPC }}$ ratio, $R$, is $\sim 0.84$ in the LE phase, and $\sim 1.73$ in the LC phase.
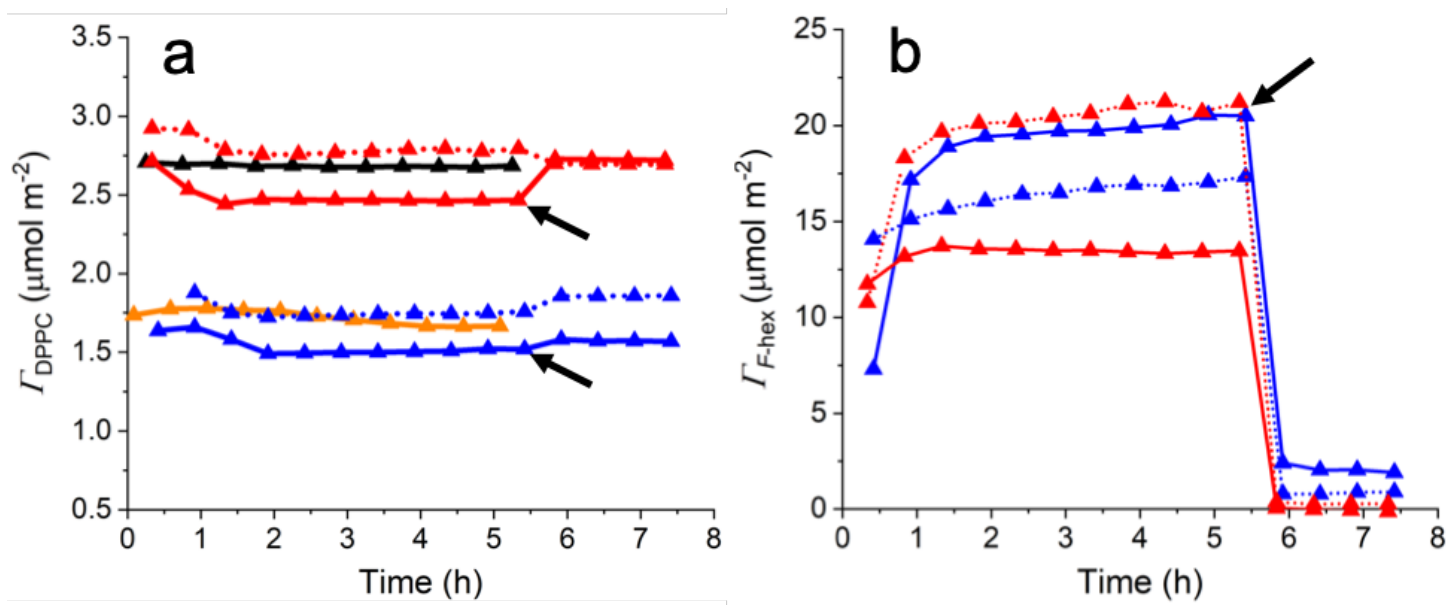

Figure 4. a) Variation of DPPC surface excesses, $\Gamma_{\mathrm{h} \text {-DPPC }}$ (dotted lines) and $\Gamma_{\mathrm{d} \text {-DPPC }}$ (solid lines) over time for monolayers in the LE phase in air (orange) and under $F$-hexane (blue), and in the LC phase in air (black) and under $F$-hexane (red). b) Variation of $\Gamma_{F-\text { hex }}$ adsorbed over time on h-DPPC (dotted line) and d-DPPC monolayers (solid line) in the LE (blue) and LC (red) phases. The arrow 
1 indicates when $F$-hexane has been vented off from the system. Errors on $\Gamma_{\mathrm{DPPC}}$ and $\Gamma_{F}$-hex were \pm 20.2 and $\pm 1.0 \mu \mathrm{mol} \mathrm{m} \mathrm{m}^{-2}$, respectively.

3 Based on the result that the surface excesses of DPPC under $F$-hexane and under air are close, as

4 calculated by co-modeling ellipsometry and NR data, the values of $\Gamma_{F \text {-hex } / \mathrm{h} \text {-DPPC }}$ and $\Gamma_{F-\text { hex } / \mathrm{d}-\mathrm{DPPC}}$,

5 and hence the values of $R$ for various surface pressures were calculated using the NR data, by fixing $6 \Gamma_{\mathrm{DPPC}}$ to its value in air. The results, collected in Figure 5, show the variation of $R$ as a function of 7 molecular area.

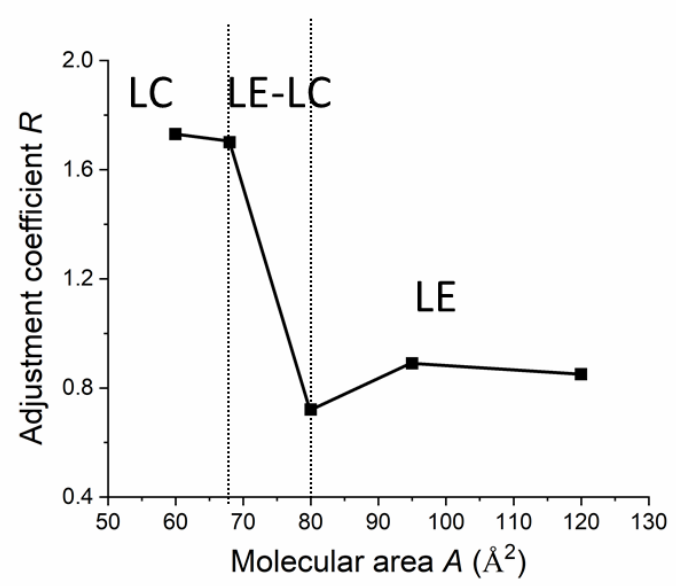

Figure 5. Variation of the adjustment coefficient $R$ for DPPC monolayers under $F$-hexane as a function of molecular area $A$.

We observe a clear trend that $R$ is dependent on the physical state of DPPC layers: in the LE phase, $R<1$, reflecting the weaker interactions developed between $F$-hexane with h-DPPC than with dDPPC; whereas in the LC phase, $R>1$, reflecting that the interactions of $F$-hexane with h-DPPC are stronger. Different strengths of $F$-hexane interactions with hydrogenous and deuterated lipids in both the LE and LC phases were thus evidenced. Deuteration results in a decrease of the intermolecular interactions among phospholipids, which is the reason why gel-to-fluid transition phase temperatures are depressed in bilayers.[67] As a consequence, $F$-hexane can more easily accommodate in d-DPPC monolayers than in h-DPPC ones in disorganized LE phase and in the coexistence region. The reverse tendency observed in the organized LC phase may be related to the fact that the interactions between C-F and C-H or C-D bonds can differ. For example, it has been found that plasticization of a semicrystalline polymer (poly(vinyl alcohol) by glycerol in the presence of a surfactant was dependent on the isotopic form of the plasticizer.[68] 
1 4. Extension to Other Phospholipids

2 In order to investigate the breath and robustness of the methodology and extend the results obtained

3 with DPPC to other systems, we investigated the interactions of $F$-hexane with two other 4 phospholipids, dimyristoylphosphatidylcholine (DMPC) and distearoylphosphatidylcholine (DSPC).

5 By contrast with DPPC, DMPC and DSPC monolayers do not experience phase transitions at room 6 temperature, and are in the LE and LC states, respectively, at the chosen surface pressure ( $35 \mathrm{mN} \mathrm{m}^{-}$

7 1; Fig. S2b,c). As for DPPC, the assumption that the fluorocarbon gas interacts similarly with the two isotopic forms led to phospholipid surface excesses that were higher than the values measured under air for the DMPC monolayers (LE phase), and lower for the DSPC monolayers (LC phase) (Fig. 6ab), which is physically unrealistic in the case of DMPC, and would mean, in the case of DSPC, that the 11 phospholipid desorbs from the surface, which is unlikely for the reasons discussed above.

12
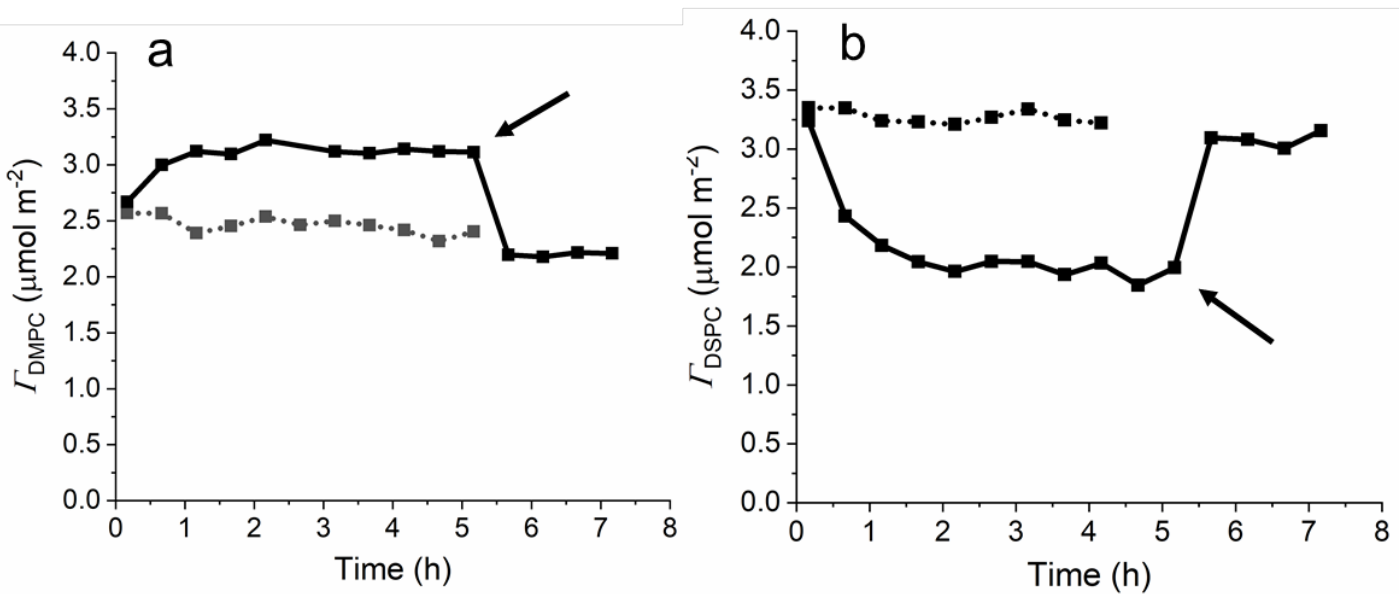

Figure 6. Variation of surface excesses $\Gamma_{\mathrm{DMPC}}$ and $\Gamma_{\mathrm{DSPC}}$ as a function of time for a) a DMPC monolayer (LE phase, $35 \mathrm{mN} \mathrm{m}^{-1}$ ) and b) a DSPC monolayer (LC phase, $30 \mathrm{mN} \mathrm{m}^{-1}$ ) under air (dotted line) and under $F$-hexane exposure (solid line). In both cases, the surface excesses are calculated with the assumption of identical interactions between $F$-hexane and the two isotopic phospholipid forms. The arrows indicate when $F$-hexane has been vented off from the system. Errors on $\Gamma_{\mathrm{DPPC}}$ were \pm $0.2 \mu \mathrm{mol} \mathrm{m}{ }^{-2}$.

We have therefore used the ratio $R$ determined in the DPPC LE phase and LC phase, respectively, that was found to be sensitive to the physical state of the monolayers to determine the adjusted surface excesses of DMPC and DSPC according to: 
$1 \quad(\rho d)_{\mathrm{d}-\mathrm{PL}}^{F-\mathrm{hex} .}=N_{\mathrm{A}}\left(\Gamma_{\mathrm{PL}} \times b_{\mathrm{d}-\mathrm{PL}}+\Gamma_{F \text {-hex } / \mathrm{d}-\mathrm{PL}} \times b_{F \text {-hex }}\right)$

Eq. 6

2 where $(\rho d)_{\mathrm{h}-\mathrm{PL}}^{F-\mathrm{hex}}$ and $(\rho d)_{\mathrm{d}-\mathrm{PL}}^{F-\mathrm{hex}}$ are the scattering excesses of h- and d-phospholipid monolayers

3 (DMPC or DSPC) exposed to $F$-hexane. $\Gamma_{\mathrm{PL}}$ is the surface excess of phospholipid. $\Gamma_{F \text {-hex/d-PL }}$ and

$4 \quad \Gamma_{F \text {-hex } / \mathrm{d} \text {-PL }}$ are the surface excesses of $F$-hexane interacting with h- or d-phospholipid.

5 The results show that, once adjusted, the phospholipid surface excesses are again close to the 6 deposited values and to the values measured under air within the experimental error $\left( \pm 0.2 \mu \mathrm{mol} \mathrm{\textrm {m } ^ { - }}\right.$ $7{ }^{2}$ ) for both DMPC and DSPC (Fig. 7ab). Enhanced interactions of $F$-hexane with d-phospholipid were 8 observed for DMPC in the LE phase, while interactions with the h-phospholipid were higher for 9 DSPC in the LC phase. These results further validate our approach based on co-modeling data of NR and ellipsometry. They also indicate that the isotopic effect of the interaction of $F$-hexane and 11 phospholipids, is strongly influenced by the phase of the monolayers, which is quantified directly 12 herein for the first time.
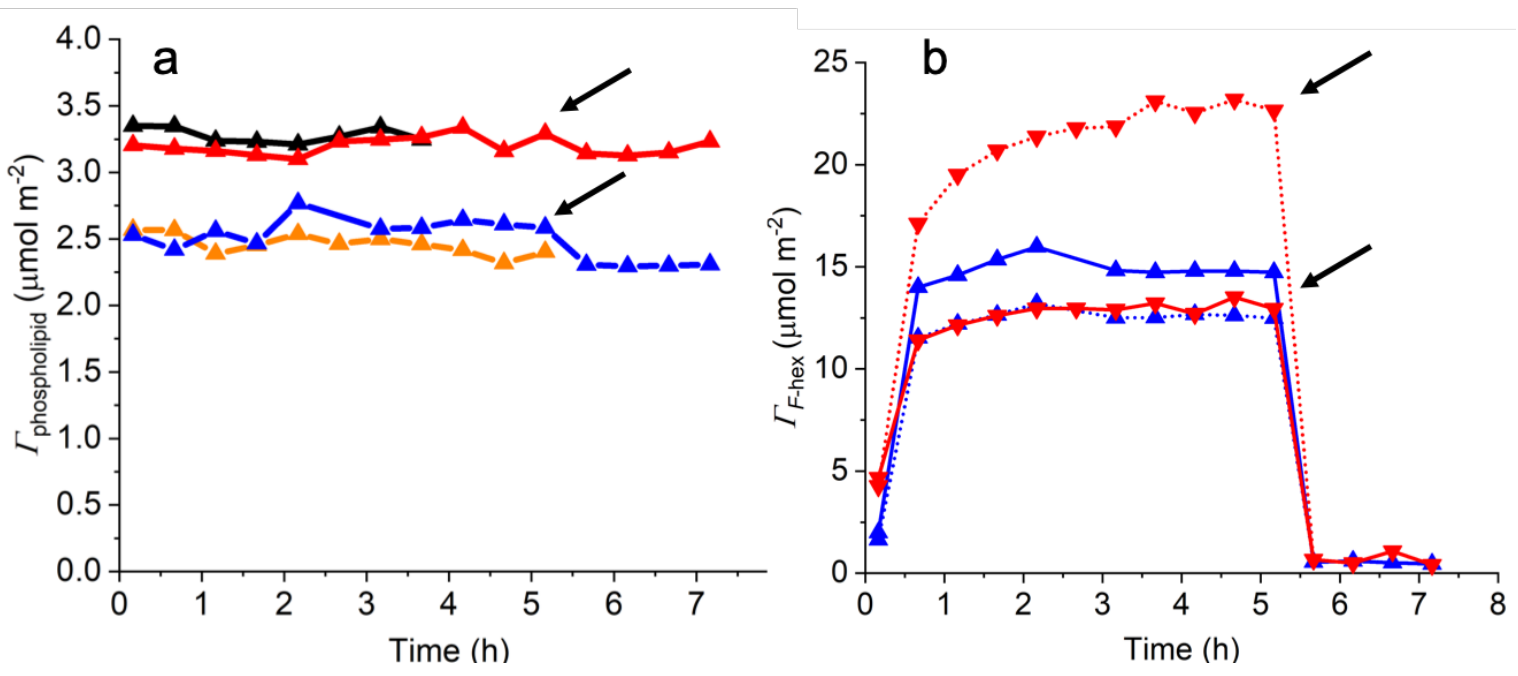

Figure 7. a) Variation of $\Gamma_{\text {DMPC }}$ over time for DMPC monolayers (LE phase) under air (orange) or under $F$-hexane (blue), and of $\Gamma_{\mathrm{DSPC}}$ for DSPC monolayers (LC phase) under air (black) or under $F$-hexane (red). b) Variation of $\Gamma_{F-\text { hex }}$ adsorbed over time on h-DMPC (blue, dotted line) and dDMPC monolayers (blue, solid line) in the LE phase; and on h-DSPC (red, dotted line) and d-DSPC monolayers (red, solid line) in the LC phase. The arrow indicates when $F$-hexane was vented off from the system. Errors on $\Gamma_{\mathrm{DPPC}}$ and $\Gamma_{F-\text { hex }}$ were \pm 0.2 and $\pm 1.0 \mu \mathrm{mol} \mathrm{m}^{-2}$, respectively. 
1 IV. CONCLUSIONS AND PERSPECTIVES

We have combined neutron reflectometry/ellipsometry data to quantify, for the first time, the extents of interaction of a fluorocarbon gas with different phospholipid monolayers with respect to their phase and isotopic contrast. This is also the first time the interfacial composition of a mixed system in which vapor adsorbs to a monolayer at the air/water interface has been resolved directly to our knowledge. The systems under investigation were DMPC, DPPC and DSPC monolayers exposed to a saturated atmosphere of $F$-hexane, and the interactions were resolved in real time.

The a priori hypothesis that the interactions of $F$-hexane with two isotopic forms of the phospholipids are the same was shown not to be valid. Differences in the extent of phospholipid monolayer/fluorocarbon gas interactions with respect to the isotopic contrast therefore could not be resolved in real time using an establish approach of neutron reflectometry alone, and instead was quantified by co-modeling neutron reflectometry and ellipsometry data. We were thus able to calculate phospholipid surface excesses during the $F$-hexane interactions that not only matched the deposited amounts but also matched the values determined under air. We also scrutinized the physical basis of the low- $Q_{z}$ analysis method of neutron reflectometry to demonstrate its suitability for purpose in the present work to a greater depth than in any other study since its development a few years ago.[28, 32] The co-modeling methodology also allowed us to quantify the strong $F$-hexane adsorption on different phospholipid monolayers. The extents of interaction depend primarily on the isotopic form of the phospholipid and on the phase of the monolayers. Monolayers of DMPC and DPPC in the liquid expanded phase experience the highest fluorocarbon adsorption when they are in deuterated form, which can be explained by the fact that the monolayers of deuterated phospholipids are less cohesive. On the other hand, monolayers of DPPC and DSPC in the liquid condensed phase experience the highest fluorocarbon adsorption when they are in their native hydrogenous form, a difference in behavior that may be related to the difference in energies of interactions between C-F and $\mathrm{C}-\mathrm{H}$ and $\mathrm{C}-\mathrm{D}$ bonds. This inference is in keeping with pronounced isotopic effects on the phase 
1 transitions in phospholipid mono- and bilayers [48, 62-65], and is a matter that warrants further

2 investigation.

3 More generally, these results open up quantification of the attraction to monolayers exerted by 4 supernatant fluorocarbon gases vis-à-vis molecular compounds such as drugs, surfactants, 5 copolymers, and others, present in the aqueous sub-phase, which has not been studied to date. We 6 envisage that this quantification will be applicable in the case of medical microbubbles, which have 7 a shell of phospholipids and are stabilized by fluorocarbons. The nature of the fluorocarbon, and hence its concentration in the bubble shell, may be key to control microbubble size and stability

9 characteristics, as well as the ability of the microbubble shell to accommodate actives and drugs. The new methodology established in the present work can therefore lead to the development and 11 optimization of emerging medical applications that has not been possible to contemplate until now.

Acknowledgements: The authors are grateful to various institutions for their financial support: the 13 French National Agency for Research (ANR, PatMol Project, Ph. D fellowship for X.L.), the 14 Strasbourg Foundation for Research in Chemistry (icFRC, Ph. D fellowship for C.C.); the 15 INTERREG V program (Nanotransmed Project; Ph. D fellowship for D.S.); the CONACYT (Mexico, 16 Ph. D fellowships for E.E.M.-O. (grant \#459199) and A.A.V.-G. (grant \# 459202). X.L., E.E.M.-O. 17 are grateful to the GIS Fluor for travel grants. We also thank the Institut Laue-Langevin for beam 18 time on FIGARO (DOI: https://doi.ill.fr/10.5291/ILL-DATA.9-13-772), as well as the Partnership 19 for Soft Condensed Matter for use of the ellipsometer.

\section{REFERENCES}

21 [1] E.S. Schutt, D.H. Klein, R.M. Mattrey, J.G. Riess, Injectable microbubbles as contrast agents for diagnostic ultrasound imaging: The key role of perfluorochemicals, Angew. Chem. Int. Ed. 42 (2003) 3218-3235.

[2] J.R. Lindner, Microbubbles in medical imaging: Current applications and future directions., Nat.

Rev. Drug Disc. 3 (2004) 527-532. 
1 [3] L. Abou-Elkacem, S.V. Bachawal, J.K. Willmann, Ultrasound molecular imaging: Moving

2 toward clinical translation, Eur. J. Radiol. 84 (2015) 1685-1693.

3 [4] S. Wang, J. Hossack, A.L. Klibanov, Targeting of microbubbles: contrast agents for ultrasound 4 molecular imaging., J. Drug Target. 26 (2018) 420-434.

5 [5] K. Graham, E. Unger, Overcoming tumor hypoxia as a barrier to radiotherapy, chemotherapy and

6 immunotherapy in cancer treatment, Intl. J. Nanomed. 13 (2018) 6049-6058.

7 [6] A. Sahu, I. Kwon, G. Tae, Improving cancer therapy through the nanomaterials-assisted 8 alleviation of hypoxia, Biomaterials 228 (2020) 119578.

9 [7] M.P. Krafft, Alleviating tumor hypoxia with perfluorocarbon-based oxygen carriers, Curr. Opin.

$10 \quad$ Pharmacol. 53 (2020) 117-125.

11 [8] M.P. Krafft, Overcoming inactivation of the lung surfactant by serum proteins: a potential role 12 for fluorocarbons?, Soft Matter 11 (2015) 5982-5994.

13 [9] D. Shi, X. Liu, C. Counil, M.P. Krafft, Fluorocarbon exposure mode markedly affects 14 phospholipid monolayer behavior at the gas/liquid interface: Impact on size and stability of 15 microbubbles, Langmuir 35 (2019) 10025-10033.

16 [10] P.N. Nguyen, T.T. Trinh Dang, G. Waton, T. Vandamme, M.P. Krafft, A nonpolar, 17 nonamphiphilic molecule can accelerate adsorption of phospholipids and lower their surface tension 18 at the air/water interface ChemPhysChem 12 (2011) 2646-2652.

19 [11] Y. Ando, H. Tabata, M. Sanchez, A. Cagna, D. Koyama, M.P. Krafft, Microbubbles with a self20 assembled poloxamer shell and a fluorocarbon inner gas, Langmuir 32 (2016) 12461-12467. 
1 [12] L. Gazzera, R. Milani, L. Pirrie, M. Schmutz, C. Blanck, G. Resnati, P. Metrangolo, M.P. Krafft,

2 Design of highly stable echogenic microbubbles through controlled assembly of their hydrophobin

3 shell, Angew. Chem. Int. Ed. 55 (2016) 10263-10267.

4 [13] G. Yang, M. O'Duill, V. Gouverneur, M.P. Krafft, Recruitment and immobilization of a 5 fluorinated biomarker across an interfacial phospholipid film using a fluorocarbon gas, Angew. Chem.

$6 \quad$ Int. Ed. 54 (2015) 8402-8406.

7 [14] C. Justeau, A.V. Vela-Gonzalez, A. Jourdan, J.G. Riess, M.P. Krafft, Adsorption of cerium salts 8 and cerium oxide nanoparticles on microbubbles can be induced by a fluorocarbon gas, ACS Sustain.

9 Chem. Eng. 6 (2018) 11450-11456.

10 [15] D. Shi, J. Wallyn, D.-V. Nguyen, F. Perton, D. Felder-Flesch, S. Bégin-Colin, M. Maaloum,

11 M.P. Krafft, Microbubbles decorated with dendronized magnetic nanoparticles for biomedical 12 imaging. Effective stabilization via fluorous interactions, Beilstein J. Nanotechnol. 10 (2019) 2103$13 \quad 2115$.

14 [16] E.E. Mendoza-Ortega, M. Dubois, M.P. Krafft, Fluorocarbon gas exposure induces 15 disaggregation of nanodiamond clusters and enhanced adsorption, enabling medical microbubble 16 formation, ACS Appl. Nano Mater. 3 (2020) 8897-8905.

17 [17] K. Ariga, T. Kunitake, Molecular recognition at air-water and related interfaces: complementary 18 hydrogen bonding and multisite interaction, Acc. Chem. Res. 31 (1998) 371-378.

19 [18] K. Ariga, T. Nakanishi, J.P. Hill, A paradigm shift in the field of molecular recognition at the 20 air-water interface: from static to dynamic, Soft Matter 2 (2006) 465-477.

21 [19] L.A. Clifton, R.A. Campbell, F. Sebastiani, J. Campos-Terán, J.F. Gonzalez-Martinez, S. 
1 interactions using complementary surface-sensitive techniques, Adv. Colloid Interface Sci. 277 (2020)

2102118.

3 [20] R. Mendelsohn, C.R. Flach, Infrared reflection-absorption spectroscopy of lipids, peptides, and

4 proteins in aqueous monolayers, Curr. Top. Membr. 52 (2002) 57-88.

5 [21] K. Kim, S.Q. Choi, J.A. Zasadzinski, T.M. Squires, Nonlinear chiral rheology of phospholipid 6 monolayers, Soft Matter 14 (2018) 2476-2483.

7 [22] D. Matyszewska, S. Moczulska, Effect of $\mathrm{pH}$ on the interactions of doxorubicin with charged 8 lipid monolayers containing 1,2-dimyristoyl-sn-glycero-3-phospho-L-serine - An important 9 component of cancer cell membranes, Electrochim. Acta 280 (2018) 229-237.

10 [23] J.R. Helliwell, Concerning the measurement of charge density X-ray diffraction data at 11 synchrotron sources: challenges and opportunities, Crystallogr. Rev. 23 (2017) 160.

12 [24] M. Elderdfi, A.F. Sikorski, Langmuir-monolayer methodologies for characterizing protein-lipid 13 interactions, Chem. Phys. Lipids 212 (2018) 61-72.

14 [25] W.M. Pazin, G.C.M. Ruiz, O.N.d.O. Jr, C.J.L. Constantino, Interaction of Artepillin C with 15 model membranes: Effects of $\mathrm{pH}$ and ionic strength, BBA - Biomembranes 1861 (2019) 410-417.

16 [26] B. Gzyl-Malcher, J. Handzlik, E. Klekowska, Temperature dependence of the interaction of 17 prazosin with lipid Langmuir monolayers, Colloids Surf. B Biointerfaces 112 (2013) 171-176.

18 [27] J.R. Lu, R.K. Thomas, J. Penfold, Surfactant layers at the air/water interface: structure and 19 composition, Adv. Colloid Interface Sci. 84 (2000) 143-304.

20 [28] L. Braun, M. Uhlig, R. von Klitzing, R.A. Campbell, Polymers and surfactants at fluid interfaces 21 studied with specular neutron reflectometry, Adv. Colloid Interface Sci. 247 (2017) 130-148. 
1 [29] T. Narayanan, H. Wacklin, O. Konovalov, R. Lund, Recent applications of synchrotron radiation

2 and neutrons in the study of soft matter, Crystallogr. Rev. 23 (2017) 160-226.

3 [30] R.A. Campbell, H.P. Wacklin, I. Sutton, R. Cubitt, G. Fragneto, FIGARO: The new horizontal neutron reflectometer at the ILL, Europ. Phys. J. Plus 126 (2011) 107.

[31] R.A. Campbell, Recent advances in resolving kinetic and dynamic processes at the air/water interface using specular neutron reflectometry, Curr. Opin. Colloid Interf. Sci. 37 (2018) 49-60.

[32] R.A. Campbell, A. Tummino, B.A. Noskov, I. Varga, Polyelectrolyte/surfactant films spread from neutral aggregates, Soft Matter 12 (2016) 5304-5312.

9 [33] A. Tummino, J. Toscano, F. Sebastiani, B.A. Noskov, I. Varga, R.A. Campbell, Effects of 10 aggregate charge and subphase ionic strength on the properties of spread polyelectrolyte/surfactant 11 films at the air/ water interface under static and dynamic conditions, Langmuir 34 (2018) 2312-2323.

12 [34] A. Angus-Smyth, R.A. Campbell, C.D. Bain, Dynamic adsorption of weakly interacting 13 polymer/surfactant mixtures at the air/water interface, Langmuir 28 (2012) 12479-12492.

14 [35] K.R. Hossain, S.A. Holt, A.P.L. Brun, H.A. Khamici, S.M. Valenzuela, X-ray and neutron 15 reflectivity study shows that CLIC1 undergoes cholesterol-dependent structural reorganization in 16 lipid monolayers, Langmuir 33 (2017) 12497-12509.

17 [36] D. Matyszewska, E. Nazaruk, R.A. Campbell, Interactions of anticancer drugs doxorubicin and 18 idarubicin with lipid monolayers: New insight into the composition, structure and morphology, J. 19 Colloid Interface Sci. 581 (2021) 403-416.

20 [37] K.C. Thompson, A.R. Rennie, M.D. King, S.J.O. Hardman, C.O.M. Lucas, C. Pfrang, B.R. 21 Hughes, A.V. Hughes, Reaction of a phospholipid monolayer with gas-phase ozone at the air-water 
1 interface: Measurement of surface excess and surface Ppessure in real time, Langmuir 26 (2010)

$2 \quad 17295-17303$.

3 [38] C. Pfrang, F. Sebastiani, C.O.M. Lucas, M.D. King, I.D. Hoare, D. Chang, R.A. Campbell,

4 Ozonolysis of methyl oleate monolayers at the air-water interface: oxidation kinetics, reaction

5 products and atmospheric implications, Phys. Chem. Chem. Phys. 16 (2014) 13220-13228.

6 [39] R.M.A. Azzam, N.M. Bashara, Ellipsometry and Polarized Light, North-Holland, New York, 71977.

8 [40] H. Motschmann, R. Teppner, Ellipsometry in Interface Science, Studies in Interface Science2001, 9 pp. $1-42$.

10 [41] J. Meunier, Light Scattering by Liquid Surfaces and Complementary Techniques, Marcel Dekker,

11 New York, 1992.

12 [42] R.A. Campbell, J.C. Ang, F. Sebastiani, A. Tummino, J.W. White, Spread films of human serum

13 albumin at the air-water interface: Optimization, morphology, and durability, Langmuir 31 (2015)

$14 \quad 13535-13542$.

15 [43] D. Ducharme, J.-J. Max, C. Salesse, R.M. Leblanc, Ellipsometric study of the physical states of 16 phosphatidylcholines at the air-water interface, J. Phys. Chem. 94 (1990) 1925-1932.

17 [44] A. Poirier, A. Banc, A. Stocco, M. In, L. Ramos, Multistep building of a soft plant protein film 18 at the air-water interface, J. Colloid Interface Sci. 526 (2018) 337-346.

19 [45] D. Beaglehole, Ellipsometric study of the surface of simple liquids, Physica 100B (1980) 16320174 
1 [46] F. Sebastiani, R.A. Campbell, C. Pfrang, Complementarity of neutron reflectometry and

2 ellipsometry for the study of atmospheric reactions at the air-water interface, RSC Adv. 5 (2015)

3 107105-107111.

4 [47] T.L. Crowley, A uniform kinematic approximation for specular reflectivity, Physica A 195 (1993)

$5 \quad 354-374$.

6 [48] R.A. Campbell, Y. Saaka, Y. Shao, Y. Gerelli, R. Cubitt, E. Nazaruk, D. Matyszewska, M.J.

7 Lawrence, Structure of surfactant and phospholipid monolayers at the air/water interface modeled

8 from neutron reflectivity data, J. Colloid Interface Sci. 531 (2018) 98-108.

9 [49] J.H. Lakey, Recent advances in neutron reflectivity studies of biological membranes, Curr. Opin.

10 Colloid Interface Sci. 42 (2019) 33-40.

11 [50] M.W.A. Skoda, Recent developments in the application of X-ray and neutron reflectivity to soft-

12 matter systems, Curr. Opin. Colloid Interface Sci. 42 (2019) 41-54.

13 [51] O. Pabois, C.D. Lorenz, R.D. Harvey, I. Grillo, M.M.-L. Grundy, P.J. Wilde, Y. Gerelli, C.A.

14 Dreiss, Molecular insights into the behaviour of bile salts at interfaces: a key to their role in lipid

15 digestion J.Colloid Interface Sci. 556 (2019) 299-277.

16 [52] M.D. Phan, O.I. Korotych, N.G. Brady, M.M. Davis, S.K. Satija, J.F. Ankner, B.D. Bruce, X17 ray and neutron reflectivity studies of styrene-maleic acid copolymer interactions with galactolipid18 containing monolayers, Langmuir 36 (2020) 3970-3980.

19 [53] A. Nelson, Co-refinement of multiple-contrast neutron/X-ray reflectivity data using MOTOFIT, 20 J. Appl. Crystallogr. 39(2) (2006) 273-276.

21 [54] A. Kabalnov, D. Klein, T. Pelura, E. Schutt, J. Weers, Dissolution of multicomponent 22 microbubbles in the blood stream: 1. Theory, Ultrasound Med. Biol. 24 (1998) 739-749. 
1 [55] M.P. Krafft, V.B. Fainerman, R. Miller, Modeling of the effect of fluorocarbon gases on the

2 properties of phospholipid monolayers and the adsorption dynamics of their aqueous solutions or 3 dispersions, Colloid Polym Sci. 293 (2015) 3091-3097.

4 [56] A.M.A. Dias, C.M.B. Gonçalves, A.I. Caço, L.M.N.B.F. Santos, M.M. Pineiro, L.F. Vega, J.A.P. 5 Coutinho, I.M. Marrucho, Densities and vapor pressures of highly fluorinated compounds, J. Chem. 6 Eng. Data 2005, 50, 50 (2005) 1328-1333.

7 [57] V.E. Stiles, G.H. Cady, Physical properties of perfluoro-n-hexane and perfluoro-28 methylpentane, J. Am. Chem. Soc. 74 (1952) 3771-3773.

9 [58] D.G. LeGrand, J. G. L. Gaines, The Polarizability of some deuterated hydrocarbons, J. Phys. 10 Chem. 98 (1994) 4842-4844.

11 [59] F. Gerber, M.P. Krafft, T.F. Vandamme, M. Goldmann, P. Fontaine, Preventing crystallization 12 of phospholipids in monolayers: a new approach to lung surfactant therapy, Angew. Chem. Int. Ed. $1344(2005) 2749-2752$.

14 [60] F. Gerber, M.P. Krafft, T.F. Vandamme, M. Goldmann, P. Fontaine, Fluidization of a 15 dipalmitoylphosphatidylcholine monolayer by fluorocarbon gases: potential use in lung surfactant 16 therapy, Biophys. J. 90 (2006) 3184-3192.

17 [61] U.K. Basak, A. Datta, Dynamics driven by lipophilic force in Langmuir monolayers: In-plane 18 and out-of-plane growth, Phys. Rev. E Stat. Nonlin. Soft Matter Phys. 91 (2015) 042405.

19 [62] N.O. Petersen, P.A. Kroon, M. Kainosho, S.I. Chan, Thermal phase transition in deuterated 20 lecithin bilayers, Chem. Phys. Lipids 14 (1975) 343-349. 
1 [63] F. Foglia, D.J. Barlow, F.C. Szoka, Z. Huang, S.E. Rogers, M.J. Lawrence, Structural studies of

2 the monolayers and bilayers formed by a novel cholesterol-phospholipid chimera, Langmuir 27 (2011) $38275-8281$.

4 [64] E. Madrid, S.L. Horswell, Effect of deuteration on phase behavior of supported phospholipid 5 bilayers: A spectroelectrochemical study, Langmuir 31 (2015) 12544-12551.

6 [65] A. Luchini, R. Delhom, B. Demé, V. Laux, M. Moulin, M. Haertlein, H. Pichler, G.A. Strohmeier,

7 H. Wacklin, G. Fragneto, The impact of deuteration on natural and synthetic lipids: A neutron 8 diffraction study, Colloids Surf. B Biointerfaces 168 (2018) 126-133.

9 [66] M. Thoma, M. Schwendler, H. Baltes, C.A. Helm, T. Pfohl, H. Riegler, H. Möhwald, 10 Ellipsometry and X-ray reflectivity studies on monolayers of phosphatidylethanolamine and 11 phosphatidylcholine in contact with n-dodecane, n-hexadecane, and bicyclohexyl, Langmuir 12 12 (1996) 1722-1728.

13 [67] G. Bryant, M.B. Taylor, T.A. Darwish, A.M. Krause-Heuer, B. Kent, C.J. Garvey, Effect of 14 deuteration on the phase behaviour and structure of lamellar phases of phosphatidylcholines 15 Deuterated lipids as proxies for the physical properties of native bilayers, Colloids Surf. B 16 Biointerfaces 177 (2019) 196-203.

17 [68] A. Briddick, P. Li, A. Hughes, F. Courchay, A. Martinez, R.L. Thompson, Surfactant and 18 plasticizer segregation in thin poly(vinyl alcohol) films, Langmuir 32 (2016) 864-872. 


\section{Supporting Information}

\section{First Quantitative Assessment of the Adsorption of a Fluorocarbon Gas on Phospholipid Monolayers at the Air/Water Interface}

Xianhe Liu, ${ }^{\text {a }}$ Claire Counil, ${ }^{\text {a }}$ Da Shi, ${ }^{\text {a }}$ Estefania E. Mendoza-Ortega, ${ }^{\text {a }}$ Andrea V. VelaGonzalez, ${ }^{a}$ Armando Maestro, ${ }^{b}$ Richard A. Campbell, ${ }^{\text {bc }}$ Marie Pierre Krafft ${ }^{a *}$

${ }^{a}$ University of Strasbourg, Institut Charles Sadron (CNRS), 23 rue du Loess, 67034 Strasbourg, France

${ }^{\mathrm{b}}$ Institut Laue-Langevin, 71 Avenue des Martyrs, CS20156, 38042 Grenoble Cedex 9, France ${ }^{\mathrm{c}}$ Division of Pharmacy and Optometry, University of Manchester, Manchester M13 9PT, United Kingdom

\section{Fitting parameter in low- $Q_{z}$ analysis}

We have chosen to fix the scattering length density $\rho$ and fit the thickness $d$ of a single uniform layer using the Motofit program in the low- $Q_{z}$ analysis in order to resolve the product $\rho d$ for each given dataset. An arbitrary value of $\rho=5 \times 10^{-6} \AA^{-2}$ was fixed, which is close to the scattering length density of d-DPPC, the highest value among the three interfacial components. An advantage of fixing a relatively high $\rho$ is that there are relatively small systematic errors introduced into the analysis. On the contrary, fixing a relatively low $\rho$ results in relatively large values of $d$, which changes the gradient of the fit and therefore augments the associated errors in $\rho d$. Examples of the surface excesses of DPPC $\left(\Gamma_{\mathrm{DPPC}}\right)$ and $F$-hexane $\left(\Gamma_{F \text {-hex }}\right)$ in the LE phase from the low- $Q_{\mathrm{z}}$ analysis by fixing this arbitrary $\rho$ value are shown in Table $\mathrm{S} 1$.

Refined values of $\rho$ for a single uniform $\mathrm{DPPC} / F$-hexane layer can be estimated from the two surface excesses determined in the optimal data analysis in the main text (Figure 7) with respect to their component molecular volumes (Table S2) using the equation $\rho_{\text {mix layer }}=\frac{\sum \rho_{\mathrm{i}} \times \Gamma_{\mathrm{i}} \times V m_{i}}{\sum \Gamma_{\mathrm{i}} \times V m_{i}}$. Values of $\Gamma_{\mathrm{DPPC}}$ and $\Gamma_{F-\text { hex }}$ obtained through application of the low- $Q_{\mathrm{z}}$ analysis when $\rho$ is fixed at the refined values, according to 
the respective isotopic contrast of DPPC, are also shown in Table S1.

Table S1: DPPC (LE phase) and $F$-hexane surface excesses $\left(\Gamma_{\mathrm{DPPC}}\right.$ and $\left.\Gamma_{F \text {-hex }}\right)$ obtained by fixing the scattering length density $\rho$ either using arbitrary or refined values. The difference associated with the two values of $\rho$ are indicated in parentheses.

\begin{tabular}{|c|c|c|c|}
\hline Mixed layers & Fitting $\rho\left(\times 10^{-6} \AA^{-2}\right)$ & $\boldsymbol{\Gamma}_{\text {DPPC }}\left(\mu \mathrm{mol} \mathrm{m}{ }^{-2}\right)$ & $\boldsymbol{\Gamma}_{F \text {-hex }}\left(\mu \mathrm{mol} \mathrm{m}{ }^{-2}\right)$ \\
\hline h-DPPC/F-hexane & \multirow{2}{*}{5 (Arbitrary) } & \multirow{2}{*}{$2.09(2.4 \%)$} & \multirow{2}{*}{$16.84(2.3 \%)$} \\
\hline d-DPPC/F-hexane & & & \\
\hline h-DPPC/F-hexane & 2.4 (Refined) & \multirow{2}{*}{2.04} & \multirow{2}{*}{17.31} \\
\hline d-DPPC/F-hexane & 4.2 (Refined) & & \\
\hline
\end{tabular}

Table S2: Values of scattering length $(b)$, molecular volume $\left(V_{\mathrm{m}}\right)$ and scattering length density $(\rho)$ used in the NR data treatment for DPPC and $F$-hexane.

\begin{tabular}{llll} 
& $b(\mathrm{fm})$ & $V_{\mathrm{m}}\left(\AA^{3}\right)$ & $\rho\left(\times 10^{-6} \AA^{-2}\right)$ \\
\hline DPPC headgroup & 60.04 & 344 & 1.75 \\
$\mathrm{~h}_{62}$-chains (LE) & -32.54 & 892 & -0.36 \\
$\mathrm{~d}_{62}$-chains (LE) & 612.98 & 892 & 6.87 \\
h-DPPC (LE) & 27.50 & 1213 & 0.23 \\
d-DPPC (LE) & 673.02 & 1213 & 5.55 \\
$F$-hexane & 117.77 & 335 & 3.52
\end{tabular}

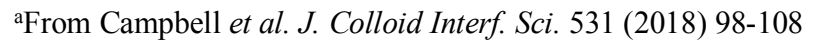

A comparison of the results using the arbitrary and refined values reveals only minimal differences in the resulting surface excesses $(<3 \%)$, which demonstrates that our approach to use an arbitrary yet relatively high value of $\rho$ in the fitting process did not introduce significant errors into the data analysis.

Furthermore, a comparison of the data analysis with fitting $\rho$ with fixed values of $d$ for the same data was tested for reference. A series of values of $d$ from 20 to $100 \AA$ were selected, and the corresponding fitted values of $\rho$ were obtained. Calculated surface excesses are shown in Table S3, as well as the differences (indicated in parentheses) associated with the values obtained with our standard fitting parameter of fixed $\rho=5 \times 10^{-6} \AA^{-2}$. 
Table S3: DPPC (LE phase) and $F$-hexane surface excesses ( $\Gamma_{\mathrm{DPPC}}$ and $\left.\Gamma_{F \text {-hex }}\right)$ obtained by fixing the thickness $d$ from 20 to $100 \AA$. The errors associated with the results obtained with fixed $\rho=5 \times 10^{-6} \AA^{-2}$ are indicated in parentheses.

\begin{tabular}{ccc}
\hline Fitting $d(\AA)$ & $\left.\boldsymbol{\Gamma}_{\text {DPPC }}(\mu \mathrm{mol} \mathrm{m})^{-2}\right)$ & $\boldsymbol{\Gamma}_{F \text {-hex }}\left(\mu \mathrm{mol} \mathrm{m}{ }^{-2}\right)$ \\
\hline 20 & $2.02(3.3 \%)$ & $16.80(0.2 \%)$ \\
\hline 40 & $2.03(2.9 \%)$ & $17.14(1.8 \%)$ \\
\hline 60 & $2.07(1.0 \%)$ & $17.69(5.0 \%)$ \\
\hline 80 & $2.14(2.4 \%)$ & $18.51(9.9 \%)$ \\
\hline 100 & $2.24(7.2 \%)$ & $19.62(16.5 \%)$ \\
\hline
\end{tabular}

It is seen that when the thickness of the layer is below $\sim 60 \AA$, the maximal error in the surface excess calculation is $\leq 5 \%$. For thicknesses larger than $\sim 60 \AA$, larger deviations in the surface excesses coincide with poorer fits to the data (Figure S1).

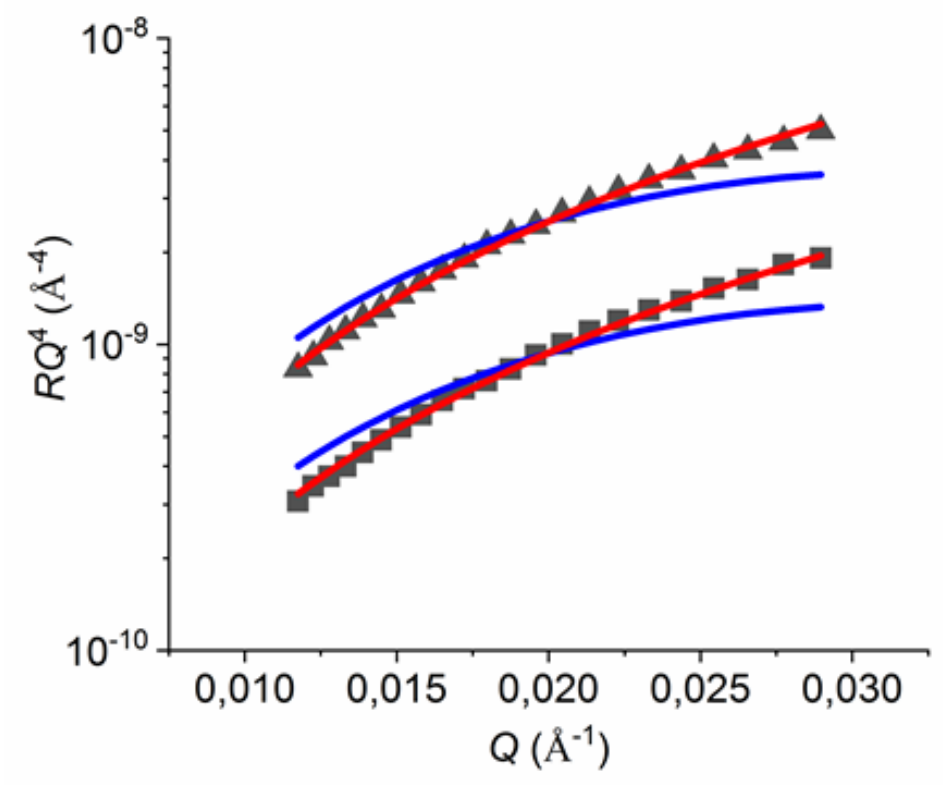

Figure S1. Variation of reflectivity $R Q^{4}$ as a function of $Q_{\mathrm{z}}$ for DPPC/F-hexane layers in the LE phase (triangles: d-DPPC; squares: h-DPPC). The fits are represented by lines using a fixed thickness $d=20 \AA$ (red) and $100 \AA$ (blue). 


\section{Surface pressure of phospholipid monolayers under air and under $F$ - hexane}

Table S4. Deposition of DPPC monolayers and corresponding phases under $F$-hexane exposure. The surface pressures reached using an adsorption trough ( $\pi$ adsorption) or Langmuir trough ( $\pi$ Langmuir) were obtained from Figures S1 and S2, respectively.

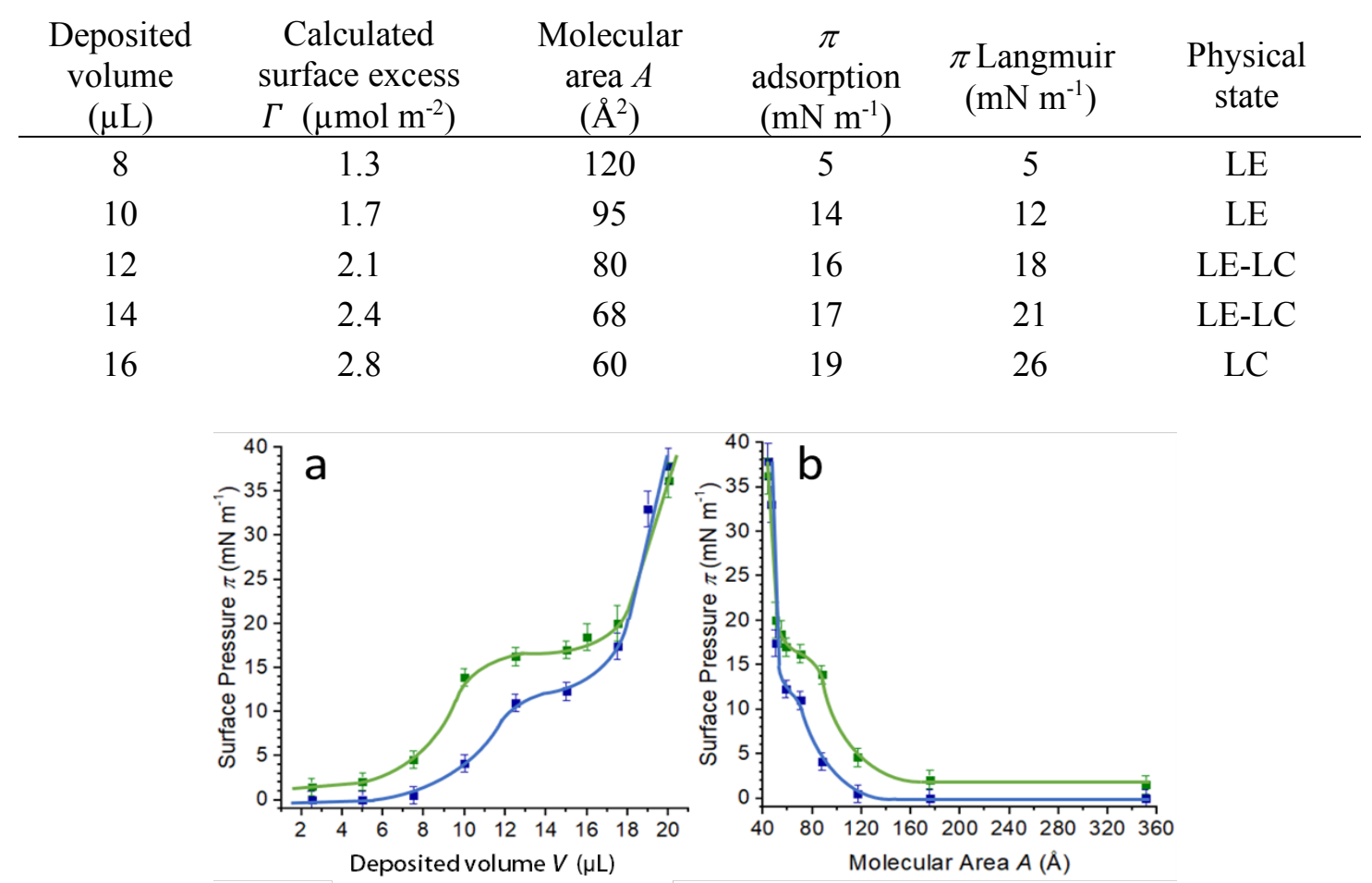

Figure S2. Variation of surface pressure $\pi$ of hydrogenous DPPC monolayers as a function of a) deposited volume of DPPC, and b) molecular area $A$ calculated from the deposited quantity. The measurements were done at $21 \pm 1^{\circ} \mathrm{C}$ under air (blue) or under $F$-hexane (green) in a trough similar to those used on FIGARO. Line are shown as a guide to the eye.
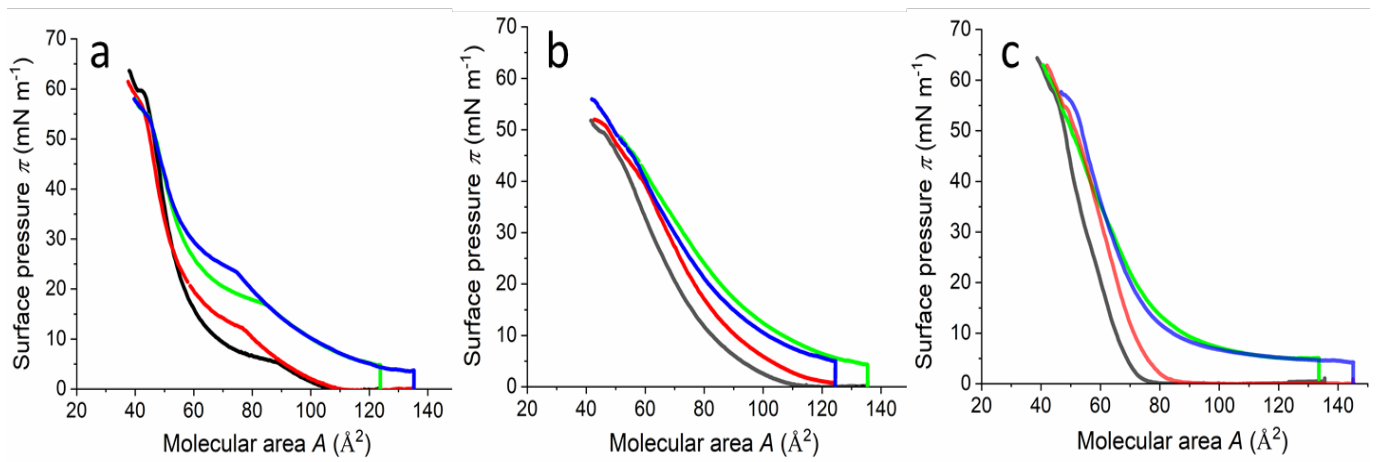

Figure S3. Variation of surface pressure $\pi$ at $21 \pm 1^{\circ} \mathrm{C}$ of Langmuir monolayers of a) DPPC, b) DMPC and c) DSPC as a function of molecular area $A$ under air or under $F$ hexane exposure. Hydrogenous and deuterated phospholipids under air are presented in black and red respectively; and in green and blue, respectively, when exposed to $F$ hexane. (NIMA trough, compression speed: $10 \mathrm{~cm}^{2} \mathrm{~min}^{-1}$ ). 


\section{Simulation of the effect of vertical separation}

Here we scrutinize whether the actual structure of phospholipid monolayers in stratified layers parallel with the air/water interface, both under air and when interacting with $F$-hexane, can result in significant uncertainties in the low- $Q_{z}$ analysis method of $\mathrm{NR}$, given that it approximates the system to single, uniform layers. We consider in turn in the following: A) a DPPC monolayer and B) its interactions with $F$-hexane.

A) DPPC molecules spontaneously organize at the air/water interface to form an upper layer occupied by the hydrophobic chains and a lower layer occupied by solvated polar headgroups, thus creating a vertical separation between two stratified layers of different scattering length densities. The question then would be: does application of the low- $Q_{z}$ analysis method to data resulting from the stratified layer structure of d-DPPC result in measurable uncertainty in the resulting surface excess given that the system is approximately to a single, uniform layer?

B) When a DPPC monolayer is exposed to $F$-hexane, the fluorocarbon molecules could, in principle, be inserted among the DPPC chains (in the same layer) or sit on top of them (forming an additional stratified layer), which indeed would result in different scattering length density profiles normal to the interface in both isotopic contrasts measured. The question would then be: does application of the low- $Q_{z}$ analysis method to these distinctly different structures in two different isotopic contrasts of the DPPC (assuming no isotope effects) result in measurably uncertainty in the resulting interfacial composition given that the system is approximately to a single, uniform layer in each isotopic contrast measured?

Simulations using three different configurations of DPPC were performed (Scheme S1, parameters in Table S5): a DPPC monolayer comprising an upper layer of chains and a lower layer of solvated headgroups (config. 1); a DPPC monolayer exposed to F- 
hexane comprising a homogeneously mixed upper layer of chains and $F$-hexane (the latter with a volume fraction of $33 \%$, DPPC thus occupying $67 \%$ of the interface) and a lower layer of solvated headgroups (config. 2); and a DPPC monolayer exposed to $F$ hexane comprising an upper layer of $F$-hexane (same surface excess as in config. 2), an intermediate layer of phospholipid chains and a lower layer of solvated headgroups (config. 3).

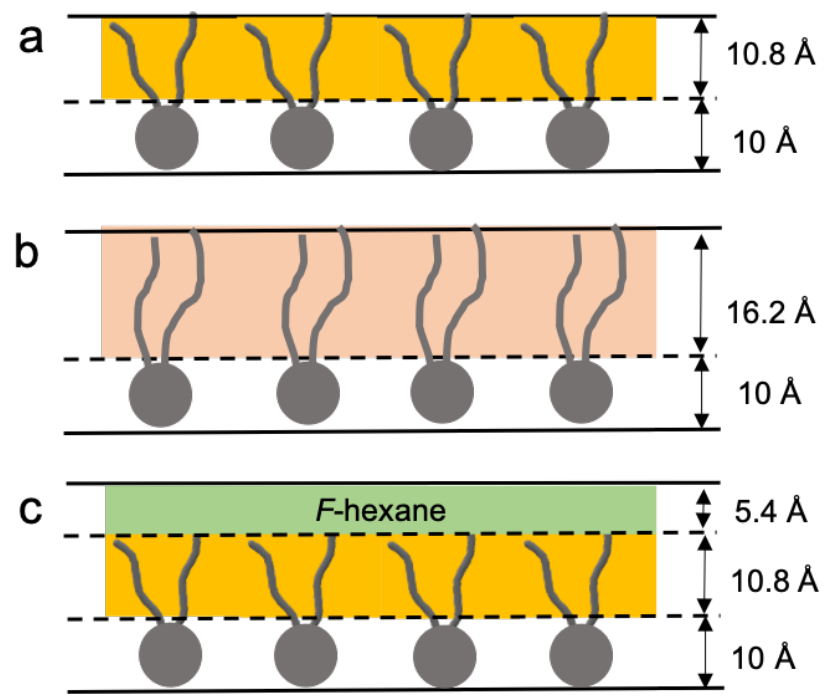

Scheme S1. Configurations of DPPC monolayers created to assess the validity of the NR data treatment using Motofit. a) Config. 1 is a DPPC monolayer exposed to air (chain region in yellow); b) config. 2 is a monolayer with $F$-hexane molecules (volume fraction 33\%) homogenously inserted within the DPPC monolayer (chain region in pink); c) config. 3 is a DPPC monolayer with a layer of $F$-hexane (green) covering the DPPC monolayer (chain region in yellow).

Table S5: Values of the parameters used for building the three configurations for DPPC monolayers (LE phase) under air or under $F$-hexane exposure.

\begin{tabular}{llll} 
& Config. 1 & Config. 2 & Config. 3 \\
\hline Chain thickness $(\AA)$ & $10.8^{\mathrm{a}}$ & 16.2 & 10.8 \\
Chain volume fraction & 1 & $0.67^{\mathrm{b}}$ & 1 \\
Headgroup thickness $(\AA)$ & $10^{\mathrm{a}}$ & 10 & 10 \\
Headgroup volume fraction & $0.42^{\mathrm{a}}$ & 0.42 & 0.42 \\
$F$-hexane thickness $(\AA)$ & - & 16.2 & 5.4 \\
$F$-hexane volume fraction & - & 0.33 & 1
\end{tabular}

aFrom Campbell et al. J. Colloid Interface Sci. 531 (2018) 98-108; b Surface coverage of DPPC under F-hexane in the LE phase $(66.7 \%)$ estimated from the ratio of the deposited volumes of DPPC under $F$-hexane and under air. 
The simulations were performed for $\mathrm{h}$ - and d-DPPC for all the configurations. The number of chains and headgroups were constrained to respect the molecular structure. For config. 1, the surface excess was then fitted to data only in the low- $Q_{z}$ region of 0.01-0.03 $\AA^{-1}$, and, for configs 2 and 3, the low- $Q_{z}$ analysis was performed on the data using the same methodology described in the main text, which is based on a single, uniform layer with respect to the isotopic contrast.

The simulated neutron reflectivity profiles from the stratified structures (parameters consistent with a full- $Q_{z}$ analysis in 4 isotopic contrasts, as presented in Campbell et al. J. Colloid Interface Sci. 531 (2018) 98-108), and the variation of the scattering length density as a function of the distance from the interface, are plotted in Figure S4. For all three configurations, excellent agreement was found between the surface excesses of the components calculated from the stratified model and that determined using the low$Q_{\mathrm{z}}$ analysis (Table S6). The errors introduced by the fit were found to be $<3 \%$ and $<6 \%$ for the layers exposed to air and $F$-hexane, respectively, that is, less than or equal to the experimental errors (7\% for DPPC and 6\% for $F$-hexane). We can therefore conclude that non-uniformity of layers in the vertical direction would introduce only minimal errors in the calculations of the DPPC and F-hexane surface excesses using our methodology. These simulations validate the use of the low- $Q_{z}$ analysis when the scattering is dominated by a strongly scattering components (e.g. d-DPPC and $F$ hexane), rendering the uncertainty introduced from the small negative scattering length of h-DPPC chains into the additivity assumption of summing the scattering contributions of the molecules at the interface as minimal. 

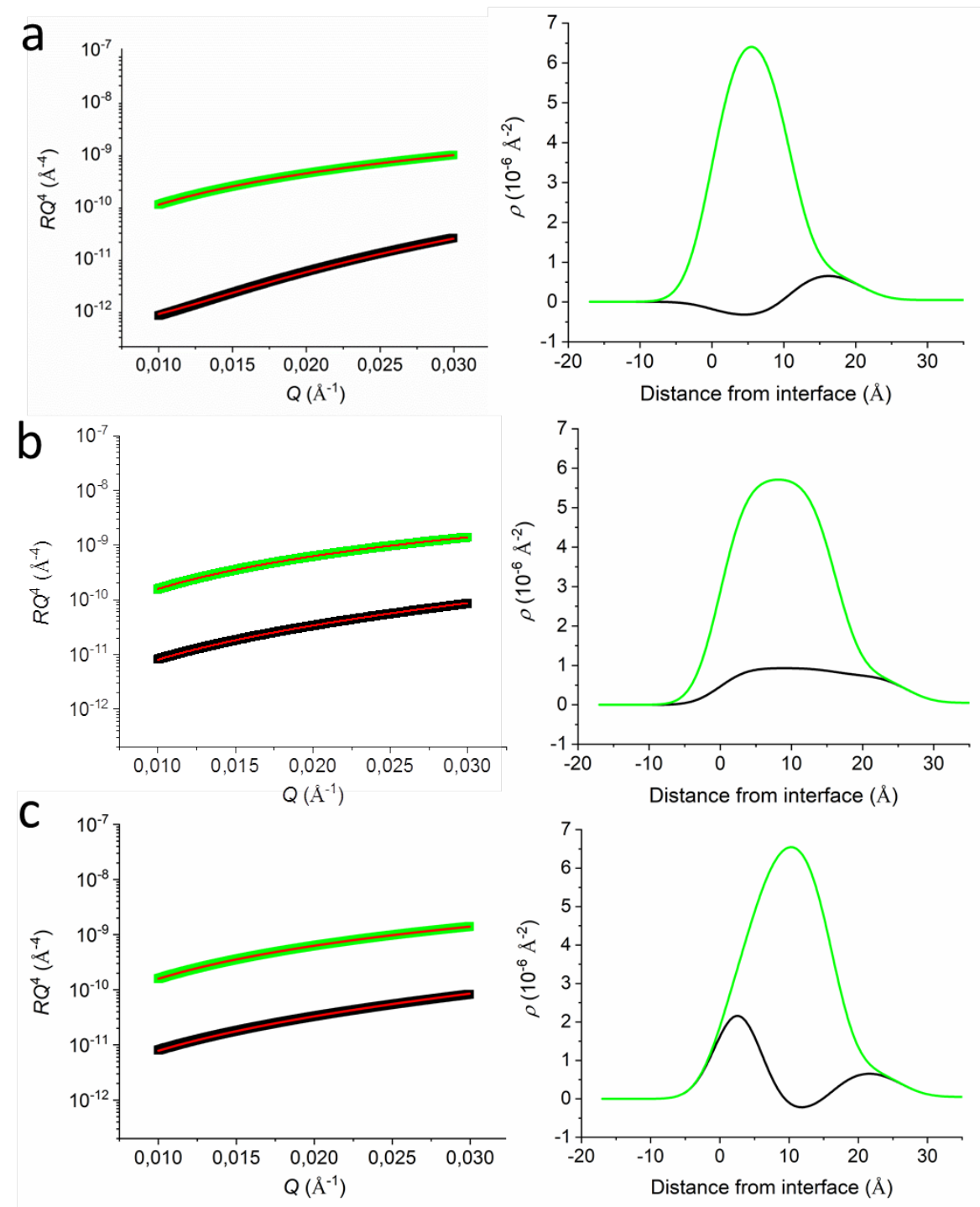

Figure S4. Left: simulated reflectivity profiles, $R Q_{\mathrm{z}}{ }^{4}$ as a function of $Q_{\mathrm{z}}$, and right: variation of scattering length density $\rho$ as a function of the distance from the interface for a) config. 1, b) config. 2, and c) config. 3 involving h-DPPC (black) and d-DPPC (green). The reflectivity is fitted with Motofit (red lines) using the low- $Q_{z}$ analysis approach by fixing a scattering length density of $5 \times 10^{-6} \AA^{-2}$ to a single uniform layer.

Table S6: Surface excesses of stratified layer structures simulated using Motofit and those calculated using the low- $Q_{\mathrm{z}}$ analysis involving a single uniform layer over the $Q_{z^{-}}$ range $0.01-0.03 \AA^{-1}$ for the three configurations examined. The errors associated with the low- $Q_{z}$ analysis method are indicated in parentheses.

Config. $1 \quad$ Config. $2 \quad$ Config. 3

\begin{tabular}{llll}
\hline Stratified $\Gamma_{\text {DPPC }}\left(\mu \mathrm{mol} \mathrm{m}{ }^{-2}\right)$ & 2.05 & 2.05 & 2.05 \\
Low $Q_{z} /$ Uniform $\Gamma_{\text {DPPC }}\left(\mu \mathrm{mol} \mathrm{m}{ }^{-2}\right)$ & $2.11(2.9 \%)$ & $2.02(1.5 \%)$ & $2.03(1.0 \%)$ \\
Stratified $\left.\Gamma_{F \text {-hex }}(\mu \mathrm{mol} \mathrm{m})^{-2}\right)$ & - & 2.67 & 2.67 \\
Low $Q_{z} /$ Uniform $\Gamma_{F-\text { hex }}\left(\mu \mathrm{mol} \mathrm{m}{ }^{-2}\right)$ & - & $2.57(3.8 \%)$ & $2.52(5.6 \%)$
\end{tabular}




\section{Simulation of the effect of lateral separation}

Here we scrutinize whether the possibility of lateral heterogeneity in the plane of phospholipid monolayers at the air/water interface when interacting with $F$-hexane can result in significant uncertainties in the low- $Q_{\mathrm{z}}$ analysis method of NR, given that it approximates the system to single, uniform layers. If lateral domains larger than the coherence length of the neutrons $(\sim 10-100 \mu \mathrm{m})$ are present, their contribution to reflectivity has to be determined separately. Modeling such a heterogeneous layer with a homogeneous model could potentially introduce significant errors into the data analysis. In order to evaluate these errors, we have fitted a mixture of DPPC and $F$ hexane at the air/water interface, both using homogeneous (scattering length density in a given layer summed with respect to their volume fraction, then a single reflectivity profile simulated) and heterogeneous (reflectivity profiles of DPPC and F-hexane simulated and then summed with respect to their area coverage) models. After these simulations were performed, the low- $Q_{\mathrm{z}}$ analysis described in the main text was carried out whilst approximating the system in each isotopic contrast to a single uniform layer. As the surface excess of $F$-hexane is larger than that of DPPC, it is impossible to have separated domains of $F$-hexane with a same height as the DPPC monolayer. The two configurations tested (Scheme S2, Table S7 for parameters used) respected the final surface excesses of $F$-hexane and DPPC determined in the present work.

As shown on Figure S5, the results show that the reflectivity is always higher for the heterogenous layer for systems involving both h-DPPC and d-DPPC. The scattering excess determined using the homogenous ( $\left.\rho d_{\text {homo }}\right)$ and heterogenous models ( $\left.\rho d_{\text {hetero }}\right)$ were obtained, and the ratio $\rho d_{\text {homo }} / \rho d_{\text {hetero }}$ was 0.5 for h-DPPC and 0.7 for $\mathrm{d}$ DPPC in the LE phase. 
a
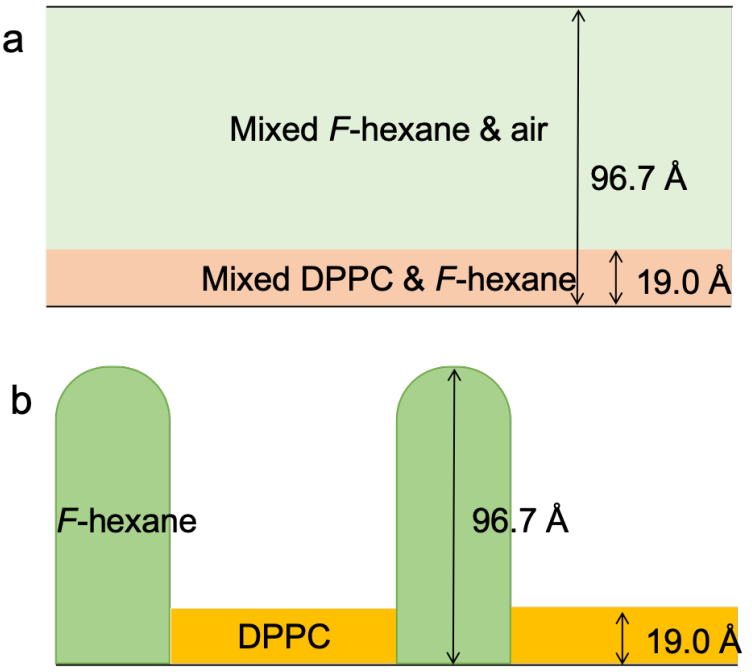

Scheme S2: Schematic representation of a) homogeneous and b) heterogeneous models corresponding to a mixture of DPPC $\left(\Gamma_{\mathrm{DPPC}} \sim 1.7 \mu \mathrm{mol} \mathrm{m}^{-2}\right)$ and $F$-hexane $\left(\Gamma_{F \text {-hex }}\right.$ $\sim 16.0 \mu \mathrm{mol} \mathrm{m}^{-2}$ ) at the air/water interface.

Table S7: Parameters used for building the heterogenous and homogenous models of DPPC monolayers under $F$-hexane (LE phase).

\section{Homogenous model}

\begin{tabular}{ll}
\hline Upper layer $(F$-hexane/air) thickness $(\AA)$ & 77.7 \\
Upper layer $F$-hexane volume fraction & 0.33 \\
Lower layer (DPPC/ $F$-hexane) thickness $(\AA)$ & 19.0 \\
Lower layer DPPC volume fraction & 0.67 \\
Lower layer $F$-hexane volume fraction & 0.33 \\
Heterogenous model & \\
\hline DPPC domain thickness $(\AA)$ & 19.0 \\
DPPC domain area coverage & 0.67 \\
$F$-hexane domain thickness $(\AA)$ & 96.9 \\
$F$-hexane domain area coverage & 0.33 \\
\hline
\end{tabular}

These ratios were thus applied to adjust the actual resolved surface excesses of DPPC under $F$-hexane using the homogeneous model to account for lateral heterogeneity in the layer structure. However, applying this correction resulted in a further increase of $\Gamma_{\mathrm{DPPC}}\left(\sim 2.7 \mu \mathrm{mol} \mathrm{m} \mathrm{m}^{-2}\right)$ to a value that is even larger than the deposited value, which is even more physically unrealistic than application of the homogenous layer model. 

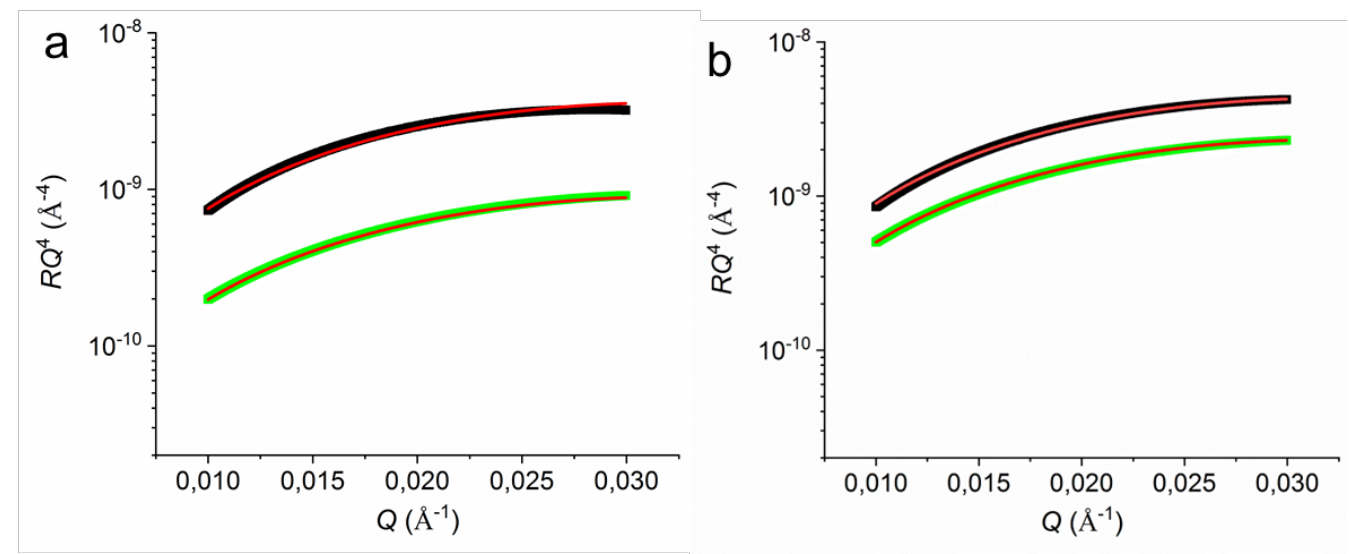

Figure S5. Simulated reflectivity profiles, $R Q_{\mathrm{z}}{ }^{4}$ as a function of $Q_{\mathrm{z}}$, for a) h-DPPC, b) d-DPPC layers mixed with $F$-hexane for the heterogenous (black) and homogenous (green) models shown in Scheme S2. The reflectivity is fitted with Motofit (red lines) using the low $-Q_{z}$ analysis approach by fixing a thickness of $100 \AA$ to a single uniform layer.

\section{Validity of the data treatment for a $45 \AA$-thick DPPC/F-hexane layer}

From the experimental design and neutron data analysis, we infer that 1) DPPC occupies $67 \%$ of the surface area in the mixed layer (a percentage coverage that corresponds to the deposited volume of the phospholipid under air and under $F$-hexane in order to reach $8 \mathrm{mN} \mathrm{m}^{-1}$ using the crude assumption of the same area per lipid molecule at the same surface pressure); 2) $F$-hexane molecules partially insert into the DPPC monolayer with the excess situated in a homogeneous layer above (note that a configuration in which full lateral separation leading to a domain thickness of $96 \AA$ has been excluded; see 'Simulation of the effect of lateral separation' above); and 3) the total thickness does not exceed $60 \AA$ given resulting poor fits of the neutron reflectivity data (see Fig. S1); 4) the total thickness of the mixed layer is equal to its nominal

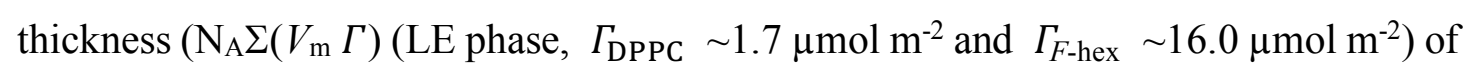
$\sim 45 \AA$ (using the assumption of conservation of volume upon mixing).

In such a configuration (Scheme S3, parameters in Table S8), an upper stratified layer consists of $F$-hexane, and a lower stratified layer consists of $F$-hexane mixed with 
DPPC (homogenously or forming lateral domains). Figure S5 shows that the homogenous (summing scattering length densities of the components by volume) or heterogenous (summing reflectivities of the domains by area coverage) character of the lower stratified layer results in minimal differences in the simulated reflectivity of the mixed layer. This estimated thickness corresponds to an upper limit of the validity of the low- $Q_{z}$ analysis of $Q_{z, \max }=1 /(45 \AA)=0.022 \AA^{-1}$. Even though the analysis was conducted on data up to $0.03 \AA^{-1}$, the experimental reflectivity profiles (Fig. 2 in main text) are in good agreement with the fitting curves over the whole $Q_{\mathrm{z}}$-range including 0.01-0.022 $\AA^{-1}$, which validates that $45 \AA$-thick DPPC/F-hexane layers can be treated using the low- $Q_{z}$ analysis method.

a

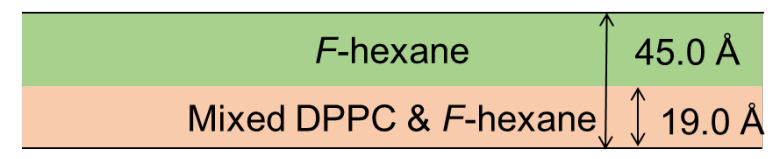

b

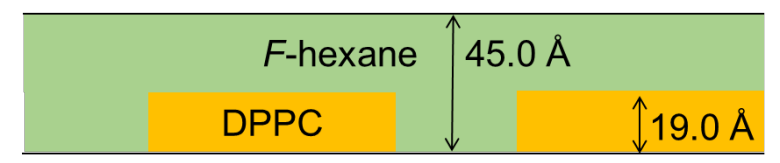

Scheme S3. Schematic representation of stratified two-layer models in which the upper layer consists of F-hexane and the lower layer consists of DPPC and $F$-hexane a) homogeneously mixed and $b$ ) heterogeneously mixed forming lateral domains. The models correspond to a mixture of DPPC $\left(\Gamma_{\mathrm{DPPC}} \sim 1.7 \mu \mathrm{mol} \mathrm{\textrm {m } ^ { - 2 }}\right)$ and $F$-hexane $\left(\Gamma_{F \text {-hex }}\right.$ $\left.\sim 16.0 \mu \mathrm{mol} \mathrm{m}^{-2}\right)$ at the air/water interface.

Table S8. Parameters used for building the homogenous and heterogenous models of DPPC monolayers under $F$-hexane (LE phase) corresponding to the two configurations depicted in Scheme S3.

\begin{tabular}{ll} 
Upper layer & \\
\hline$F$-hexane thickness $(\AA)$ & 26.0 \\
$F$-hexane volume fraction & 1 \\
\hline Lower layer & 19.0 \\
\hline DPPC/F-hexane thickness $(\AA)$ & 0.67 \\
DPPC volume fraction (homogeneous) or domain area coverage (heterogenous) & 0.33 \\
\hline -hexane volume fraction (homogeneous) or domain area coverage (heterogenous) & 0.
\end{tabular}



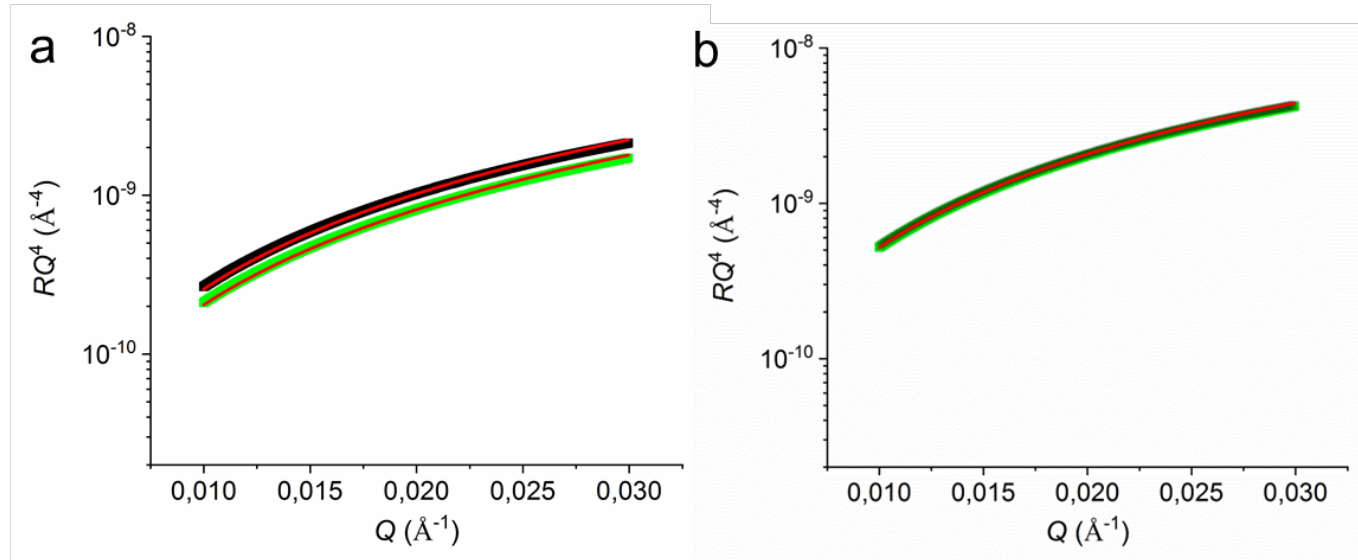

Figure S6. Simulated reflectivity profiles, $R Q_{\mathrm{z}}{ }^{4}$ as a function of $Q_{\mathrm{z}}$, for a) h-DPPC and b) d-DPPC layers mixed with $F$-hexane for the two configurations shown in Scheme S3: $a$ (green) and $b$ (black). The differences between the two configurations are minimal for h-DPPC and negligible for d-DPPC. The reflectivity is fitted with Motofit (red lines) using the low- $Q_{z}$ analysis approach by fixing a scattering length density of $5 \times 10^{-6} \AA^{-2}$ to a single uniform layer. 